ARTICLE

https://doi.org/10.1038/s41467-019-11547-5

\title{
MpsAB is important for Staphylococcus aureus virulence and growth at atmospheric $\mathrm{CO}_{2}$ levels
}

Sook-Ha Fan ${ }^{1,5}$, Patrick Ebner ${ }^{1,5}$, Sebastian Reichert ${ }^{1}$, Tobias Hertlein ${ }^{2}$, Susanne Zabel ${ }^{3}$, Aditya Kumar Lankapalli ${ }^{4}$, Kay Nieselt $\mathbb{B}^{3}{ }^{3}$, Knut Ohlsen ${ }^{2}$ \& Friedrich Götz ${ }^{1}$

The mechanisms behind carbon dioxide $\left(\mathrm{CO}_{2}\right)$ dependency in non-autotrophic bacterial isolates are unclear. Here we show that the Staphylococcus aureus mpsAB operon, known to play a role in membrane potential generation, is crucial for growth at atmospheric $\mathrm{CO}_{2}$ levels. The genes mpsAB can complement an Escherichia coli carbonic anhydrase (CA) mutant, and $C A$ from $E$. coli can complement the $S$. aureus delta-mps $A B C$ mutant. In comparison with the wild type, $S$. aureus mps mutants produce less hemolytic toxin and are less virulent in animal models of infection. Homologs of mps $A$ and $m p s B$ are widespread among bacteria and are often found adjacent to each other on the genome. We propose that MpsAB represents a dissolved inorganic carbon transporter, or bicarbonate concentrating system, possibly acting as a sodium bicarbonate cotransporter.

\footnotetext{
${ }^{1}$ Microbial Genetics, Interfaculty Institute of Microbiology and Infection Medicine Tübingen (IMIT), University of Tübingen, Auf der Morgenstelle 28, D72076 Tübingen, Germany. ${ }^{2}$ Institute for Molecular Infection Biology (IMIB), University of Würzburg, Josef-Schneider-Strasse 2, D-97080 Würzburg, Germany. ${ }^{3}$ Center for Bioinformatics Tübingen, University of Tübingen, Sand 14, D-72076 Tübingen, Germany. ${ }^{4}$ Department of Archaeogenetics, Max Planck Institute for the Science of Human History, Kahlaische Strasse 10, D-07745 Jena, Germany. ${ }^{5}$ These authors contributed equally: Sook-Ha Fan, Patrick Ebner. Correspondence and requests for materials should be addressed to F.G. (email: friedrich.goetz@uni-tuebingen.de)
} 
arly concepts of the function of $\mathrm{CO}_{2}$ in bacterial growth can - be traced back to as early as a century ago. However, convincing experimental evidence only surfaced in 1935 when Gladstone reported that $\mathrm{CO}_{2}$ is an essential factor in the growth of bacteria ${ }^{1}$. The growth-promoting effect of $\mathrm{CO}_{2}$ was observed in various bacteria, including Staphylococcus aureus, particularly when glucose was substituted by another carbon source. Many years later, $\mathrm{CO}_{2}$-dependent $S$. aureus was isolated from abscesses and osteomyelitis ${ }^{2,3}$. These strains, though very rare, were well documented as dwarf or gonidial $(G)$ strains and usually produced tiny colonies in the absence of additional $\mathrm{CO}_{2}{ }^{4}$. Although $\mathrm{G}$ strains can be isolated from lesions, their virulence is low in a mouse infection model ${ }^{3}$. Since the early 1970's, the $\mathrm{CO}_{2}$ dependence of staphylococci has been largely overlooked due in part to the routine cultivation of clinical isolates in $\mathrm{CO}_{2}$ incubators.

Renewed interest in these so-called dwarf colonies, now referred to as small colony variants ( $\mathrm{SCVs}$ ), was initiated in large part because they were frequently associated with persistent and relapsing infections such as airway infections and osteomyelitis ${ }^{5-7}$. In most cases, SCVs consist of mutants that are frequently affected in respiration comprising thymidine, hemin, or menadione auxotrophs ${ }^{8-10}$. However, unlike the earlier dwarf mutants described by Slifkin ${ }^{3}$ the respiratory mutants cannot be complemented by elevated $\mathrm{CO}_{2}$. There are several reports where $\mathrm{CO}_{2}$-dependent SCVs of $S$. aureus have been isolated from patients with catheter-related bacteremia, endocarditis, wound infections, respiratory infections, or nasal colonization ${ }^{11}$. This shows that $\mathrm{CO}_{2}$-dependent SCVs play a role in certain infections and further studies are necessary to unravel the genetic and biochemical basis of this type of auxotrophy.

We previously investigated whether $S$. aureus possesses a type 1 electrogenic NADH:quinone oxidoreductase (Ndh1), a proton pump that translocates cations, such as $\mathrm{H}^{+}$or $\mathrm{Na}^{+}{ }^{12}$. It turned out that such an Ndh1 is not present in staphylococci; however, we found S. aureus has a homolog of the Escherichia coli specific NuoL protein, one of the core components of the proton pumping mechanism in E. coli ${ }^{13}$. We named the gene $m p s A$ (membrane potential-generating system), because an $m p s A$ mutant was severely defected in membrane potential generation and also grew like an $\mathrm{SCV}^{12}$. This connection and in light of the recent attention on SCVs, we sought to determine the function of the mps operon.

In $S$. aureus, $m p s A$ is cotranscribed with the $m p s B$ gene, and is separated by a weak transcription terminator from the downstream $m p s C$. It was unknown at that time whether $m p s C$ contributes to the MpsAB function. MpsA, like the NuoL homolog is a membrane protein with predicted 14 transmembrane domains, while $\mathrm{MpsB}$ is a cytoplasmic protein with a predicted metal binding site. While MpsABC lacks NADH oxidation activity, it participates in $\mathrm{Na}^{+}$transport as mpsABC complemented an $\mathrm{Na}^{+} / \mathrm{H}^{+}$antiporter-deficient $E$. coli strain, suggesting that MpsABC constitutes a cation-translocating system ${ }^{12}$.

Here, we extend our previous observations by analyzing the function of MpsABC in greater detail. In principle, this study builds on the work of the 1970s, in which dwarf colonies were found to be complemented by $\mathrm{CO}_{2}$. We show that MpsAB increase the uptake of bicarbonate and represents a new class of dissolved inorganic carbon (DIC) transporter. DIC transporters are responsible for creating an elevated concentration of intracellular bicarbonate and supplying it to carboxysomes and Rubisco. Such transporters are most well studied in cyanobacteria $^{14}$, but are not fully described in other autotrophic bacteria. In $S$. aureus, SCV-like $m p s A B$ mutants cannot supply and store sufficient bicarbonate from atmospheric air to feed the important carboxylase reactions in central metabolism. The severe growth defect can only be compensated by increased $\mathrm{CO}_{2} /$ bicarbonate supplementation. Importantly, MpsAB homologs are widespread in bacteria and were recently found in autotrophic bacteria functioning as DIC transporters to supply carboxysomes and RubisCO with bicarbonate/ $\mathrm{CO}_{2}{ }^{15,16}$.

\section{Results}

Growth defect of $\triangle m p s A B C$ is rescued by elevated $\mathrm{CO}_{2} /$ $\mathrm{NaHCO}_{3}$. As previously reported, deletion of the $m p s A B C$ operon in $S$. aureus strain SA113 resulted in a severe growth defect, with the typical SCV-like phenotype ${ }^{12}$. To further characterize this operon, we constructed single deletion mutants of $\triangle m p s A, \triangle m p s B$, and $\triangle m p s C$, as well as a deletion of the entire operon, $\triangle m p s A B C$, in the agr-positive $S$. aureus strain $\mathrm{HG} 001^{17}$. The mutants were analyzed for growth in liquid culture under atmospheric growth conditions. As shown in Fig. 1a, the mutants $\triangle m p s A, \triangle m p s B$, and $\triangle m p s A B C$ grew slowly and did not achieve the final OD of the wild type (WT) strain HG001, even after $72 \mathrm{~h}$. The growth delay was most pronounced in the first $24 \mathrm{~h}$. In contrast to $\triangle m p s A$ and $\triangle m p s B$, the growth of $\triangle m p s C$ was not affected, indicating that $m p s C$ does not contribute to the $m p s A B$ activity.
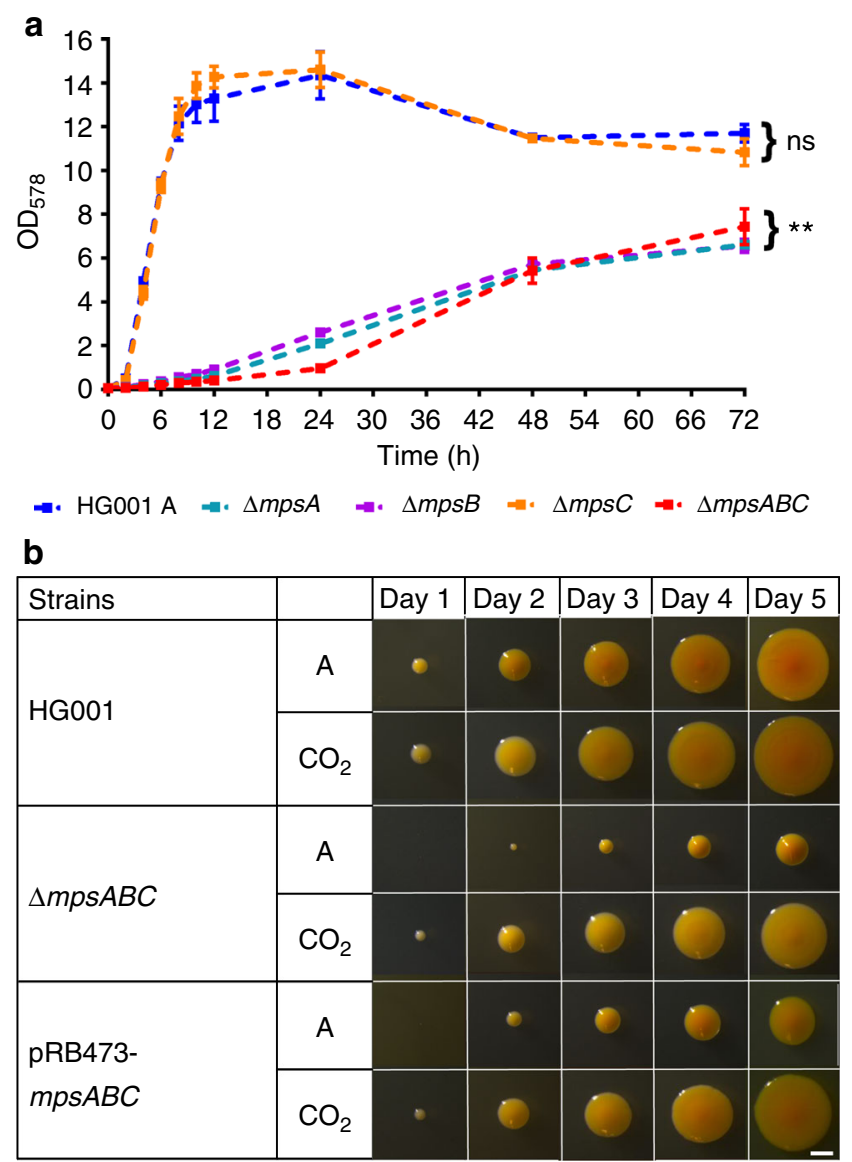

Fig. 1 Growth of HG001 $\triangle m p s A B C$. a Growth of wild type HG001, $\triangle m p s A$, $\triangle m p s B, \triangle m p s C$, and $\triangle m p s A B C$ in atmospheric (A) conditions. The growth of all mutants except $\triangle m p s C$ was significantly lower compared to HG001, ${ }^{\star \star} p<0.01$ by unpaired two-sided $t$-test. $\triangle m p s C$ showed no significant difference (ns) in growth compared to HG001. Each point shown in the graph represents the mean value \pm standard error mean (SEM) from three independent experiments. Source data are provided as a Source Data file. b Colony appearances of wild type HG001, $\triangle m p s A B C$, and its complemented mutant (plasmid pRB473-mpsABC) during 5 days of incubation in atmospheric $(A)$ conditions and $5 \% \mathrm{CO}_{2}$. White bar represents a scale of $1 \mathrm{~mm}$ 


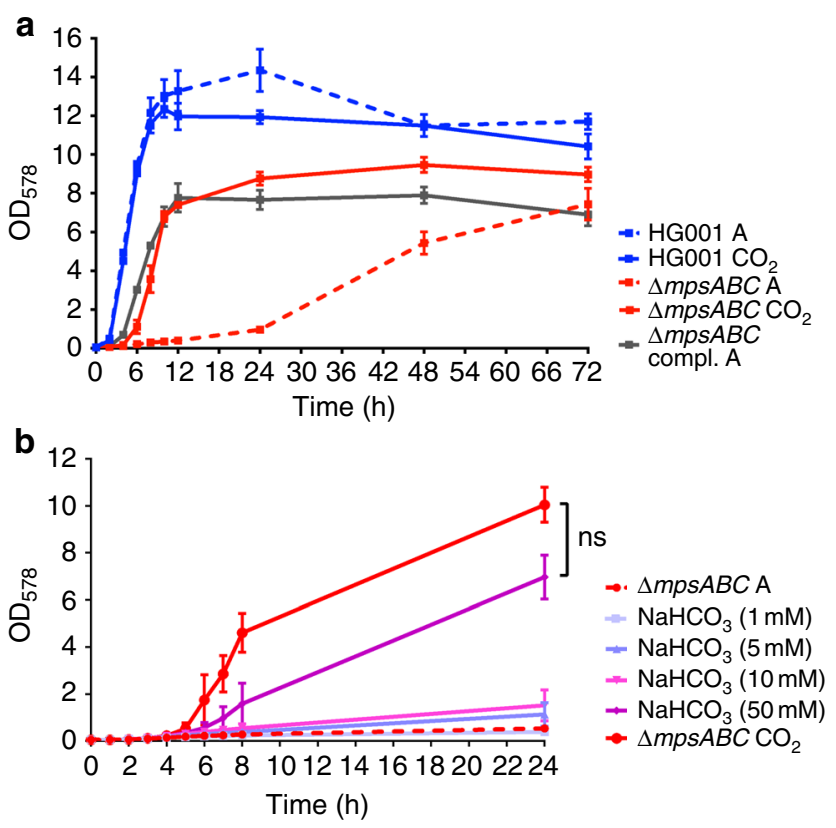

Fig. 2 Growth of HG001 $\triangle m p s A B C$ can be complemented with $5 \% \mathrm{CO}_{2}$ and $\mathrm{NaHCO}_{3}$. a Growth of $\mathrm{HGO01}, \triangle m p s A B C$ in atmospheric (A)conditions and $5 \% \mathrm{CO}_{2}$. The growth of the mutant can also be complemented by plasmid pRB473 carrying mpsABC (compl.). Each point shown in the graph represents the mean value \pm standard error mean (SEM) from three independent experiments. b Growth of $\triangle m p s A B C$ in atmospheric $(A)$ and with additions of $\mathrm{NaHCO}_{3}$ ranging from concentrations of 1 to $50 \mathrm{mM}$, and $5 \% \mathrm{CO}_{2}$. The growth of $\triangle m p s A B C$ with addition of $50 \mathrm{mM} \mathrm{NaHCO}_{3}$ was similar to that under $5 \% \mathrm{CO}_{2}$, as there was no significance difference (ns) between the two conditions ( $p>0.05$ ) by unpaired two-sided $t$-test. Each point shown in the graph represents the mean value \pm standard error mean (SEM) from two independent experiments. Source data are provided as a Source Data file

Interestingly, growth of all mutants could be restored to near WT levels under elevated $(5 \%) \mathrm{CO}_{2}$ concentrations. During the course of 5 days incubation in $5 \% \mathrm{CO}_{2}$, the colonies of all mutants were significantly larger than those grown in atmospheric air. Growth of the WT strain was largely uninfluenced by $\mathrm{CO}_{2}$, although the colony size was slightly increased (Fig. $1 \mathrm{~b}$ and Supplementary Fig. 1). The growth-promoting effect of $\mathrm{CO}_{2}$ was also seen in liquid medium (Fig. 2a and Supplementary Fig. 2). Comparison of the growth in tryptic soy broth (TSB) in the absence or presence of $5 \% \mathrm{CO}_{2}$ revealed that growth of the $\triangle m p s A B C$ mutant was significantly improved in the presence of $\mathrm{CO}_{2}$ (Fig. 2a). This growth tendency is similar in the complemented mutant $\triangle m p s A B C(\mathrm{pRB} 473-m p s A B C)$ under atmospheric conditions. Given that $\mathrm{CO}_{2}$ and bicarbonate are interconvertible in aqueous solution, we also attempted to complement $\triangle m p s A B C$ with sodium bicarbonate $\left(\mathrm{NaHCO}_{3}\right)$. $\mathrm{NaHCO}_{3}$ at concentration ranging from 1 to $50 \mathrm{mM}$ were added to the growth medium and growth of $\triangle m p s A B C$ was followed for $24 \mathrm{~h}$. At low concentrations $\left(1 \mathrm{mM} \mathrm{NaHCO}_{3}\right)$ no difference in the growth of $\triangle m p s A B C$ was observed (Fig. 2b). However, with increasing concentrations of up to $50 \mathrm{mM} \mathrm{NaHCO}$, its growth increased steadily before reaching final OD values comparable with those of $5 \% \mathrm{CO}_{2}$. Similar observation was also seen with $\triangle m p s B$ (Supplementary Fig. 3a and $3 \mathrm{~b}$ ). The $\mathrm{pH}$ of the medium (TSB) increased with the addition of $\mathrm{NaHCO}_{3}$, however the effect of $\mathrm{pH}$ of the medium did not play a crucial role on the growth of $\triangle m p s A B C$ (see Supplementary Note 1, Supplementary Table 3 and Supplementary Fig. 8). The results indicate that the growth deficiency of $\triangle m p s A B C$ can be largely compensated by the addition of $5 \% \mathrm{CO}_{2}$ and high concentrations of $\mathrm{NaHCO}_{3}$.

MpsABC increase the uptake of bicarbonate. The diminished ability of the $\triangle m p s A B C$ mutant to grow under atmospheric conditions as well as the rescue of this defect by $\mathrm{CO}_{2}$ and bicarbonate led us to the suggestion that MpsAB may play a role in $\mathrm{CO}_{2}$ and bicarbonate transport. As the determination of the $\mathrm{CO}_{2}$ transport is very difficult, we compared the bicarbonate uptake activity in the WT strain HG001 and the $\triangle m p s A B C$ mutant with radiolabeled $\mathrm{NaH}^{14} \mathrm{CO}_{3}$. Both strains were grown aerobically in TSB until reaching its respective exponential growth phase before being washed to remove the growth media and resuspended to the same $\mathrm{OD}_{578}$ in Tris buffer. Prior to the addition of $\mathrm{NaH}^{14} \mathrm{CO}_{3}$, the cell suspensions were incubated with fluorocitrate and glucose. Fluorocitrate, an aconitase inhibitor, was added to block the TCA cycle so that $\mathrm{NaH}^{14} \mathrm{CO}_{3}$ accumulated intracellularly and is not immediately expired by the decarboxylation reactions of the TCA cycle. Glucose was added as energy source because the cells were resuspended in only Tris buffer. After addition of $\mathrm{NaH}^{14} \mathrm{CO}_{3}$, samples were rapidly harvested at several time points and the ${ }^{14} \mathrm{C}$ uptake was measured by liquid scintillation counting. The immediate uptake occurred within $120 \mathrm{~s}$ until a steady state level was reached (Fig. 3a). Compared with the WT strain HG001, $\triangle m p s A B C$ showed significantly decreased ${ }^{14} \mathrm{C}$ uptake in the first $120 \mathrm{~s}$. After $15 \mathrm{~min}$, $\triangle m p s A B C$ showed a 6.6 -fold lower radioactivity than the WT strain indicating that the bicarbonate uptake was impaired in the mutant.

$m p s A B$ complements an $E$. coli $C A$ mutant and vice versa. Previously we described that MpsB contains a metal binding motif (His607, Cys622, and Cys625), which is reminiscent of a carbonic anhydrase (CA) motif ${ }^{12}$. Therefore, we tried to detect CA activity with purified MpsB alone and cell extracts expressing MpsABC. However, we were unable to detect any CA activity with various methods. In the absence of any detectable enzymatic data, we asked if $m p s A B$ could complement a $\mathrm{CO}_{2}$-dependent $E$. coli mutant, EDCM636 ${ }^{18}$. EDCM636 is an E. coli K-12 mutant in which the CA gene, can, was deleted. As a result, this strain is unable to grow under atmospheric air, but can grow in the presence of high $\mathrm{CO}_{2}$. This phenotype of EDCM636 is similar to that of HG001 $\triangle m p s A B C$; hence, we investigated whether $m p s A B$ can restore growth of EDCM636 under atmospheric conditions. To do this, we inserted the $S$. aureus $m p s A, m p s B, m p s A B$, and $m p s A B C$ genes into $E$. coli strain EDCM636. The vector alone, pRB473, served as control. Interestingly, neither $m p s A$ nor $m p s B$ alone could restore growth of EDCM636. Plasmids containing $m p s A B$ and $m p s A B C$ were able to restore the growth equally well (Fig. 4a), indicating that $m p s C$ was dispensable. As expected, all six strains were able to grow under $5 \% \mathrm{CO}_{2}$ conditions (Fig. 4a).

Since $m p s A B$ restored the growth of EDCM636, we asked if the CA gene (can) from E. coli could complement the $S$. aureus $\triangle m p s A B C$. To address this question, can from E.coli K-12 was expressed in $\triangle m p s A B C(\mathrm{pTX} 30-c a n)$ under a xylose inducible promoter. As shown in Fig. 4b, without xylose $\triangle m p s A B C$ (pTX30can) could not grow under atmospheric air. However, when can expression was induced by xylose, growth was restored. All clones grew in the presence of $5 \% \mathrm{CO}_{2}$ (Fig. $4 \mathrm{~b}$ ), suggesting that both the bicarbonate transporter and $\mathrm{CA}$ enable growth under atmospheric $\mathrm{CO}_{2}$ level and both are interchangeable. Although the activities of bicarbonate transporters and CA are different, they represent a $\mathrm{CO}_{2} /$ bicarbonate concentrating system that can functionally replace each other. 


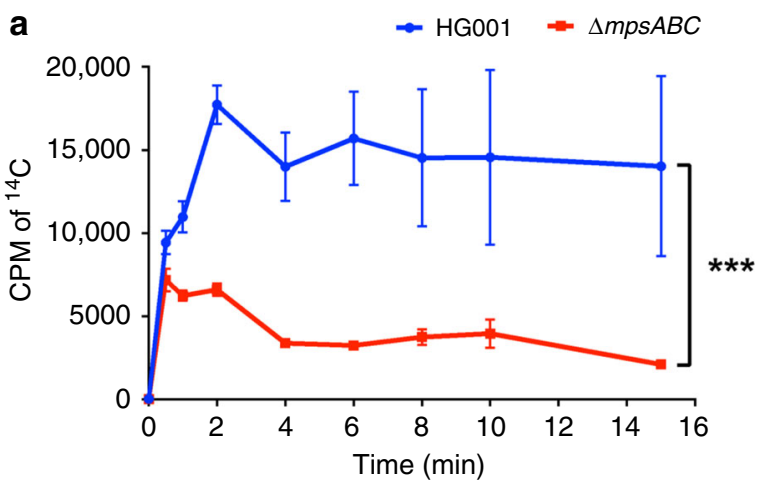

b

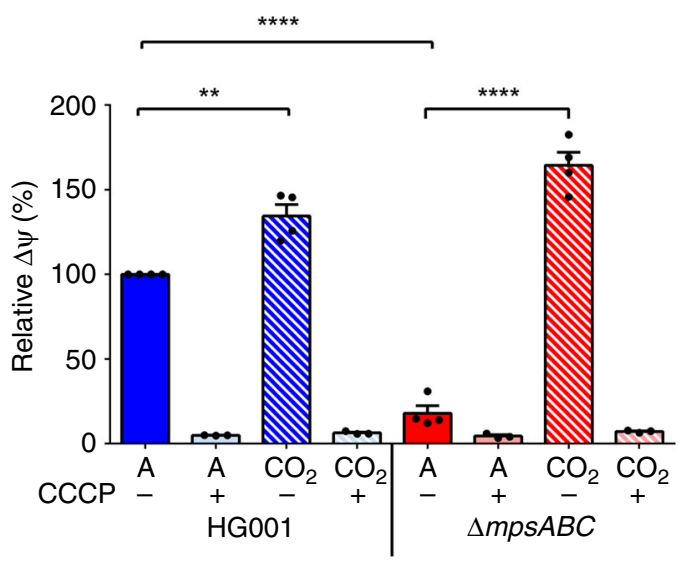

C

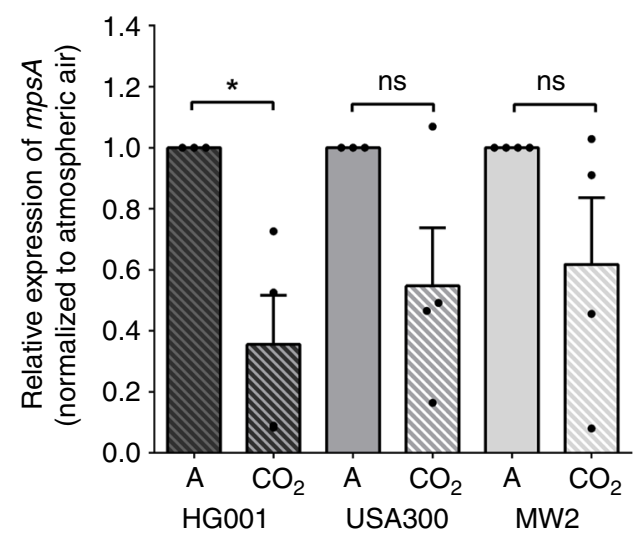

Impaired membrane potential in $\triangle m p s A B C$ is rescued by $5 \%$ $\mathbf{C O}_{2}$. Previously, it was reported that MpsABC represents a membrane potential-generating and cation-translocating system, and that membrane potential was severely affected in the $\triangle m p s A B C$ mutant ${ }^{12}$. Because elevated $\mathrm{CO}_{2}$ restores the growth of $\triangle m p s A B C$, we wondered whether $5 \% \mathrm{CO}_{2}$ also restores the membrane potential in this mutant. The membrane potential of the WT strain, HG001, grown under atmospheric air was set equal to $100 \%$ and was used as a comparator for other strains (Fig. $3 \mathrm{~b}$ ). In the $\triangle m p s A B C$ mutant, the membrane potential was decreased to only $18 \%$ of HG001. In contrast, the presence of $5 \%$ $\mathrm{CO}_{2}$ increased the membrane potential of the $\triangle m p s A B C$ mutant and the WT strain up to $164 \%$ and $134 \%$, respectively. As a negative control, the $\mathrm{H}^{+}$ionophore CCCP caused a collapse of the membrane potential in all strains tested both in atmospheric $\mathrm{CO}_{2}$ and in the presence of $5 \% \mathrm{CO}_{2}$. Based on these findings, elevated $\mathrm{CO}_{2}$ not only restores growth but also the membrane potential generation in the $\triangle m p s A B C$.
Fig. 3 Uptake of $\mathrm{NaH}^{14} \mathrm{CO}_{3}$ by $\mathrm{HGOO1}$ and $\triangle m p s A B C$, membrane potential determination and mpsA expression. a Cells were preincubated with fluorocitrate for 30 mins prior to the addition of $\mathrm{NaH}^{14} \mathrm{CO}_{3}$ $(50 \mu \mathrm{Ci}) . \mathrm{NaH}^{14} \mathrm{CO}_{3}$ was added at time 0 and $1 \mathrm{ml}$ of cell samples collected at times indicated. The $\mathrm{H}^{14} \mathrm{CO}_{3}$ uptake was determined by ${ }^{14} \mathrm{C}$ accumulation in cells measured by liquid scintillation counting. The $\mathrm{NaH}^{14} \mathrm{CO}_{3}$ uptake in $\triangle m p s A B C$ was significantly lower than WT HGO01; ${ }^{\star \star \star} p<0.001$ by paired two-sided $t$-test. Each point shown in the graph represents the mean value \pm standard error mean (SEM) from three independent experiments. ( $C P M=$ counts per minute). b $\triangle m p s A B C$ grown in $5 \% \mathrm{CO}_{2}$ showed increased membrane potential compared with those grown in atmospheric conditions. Membrane potential was determined by fluorescence intensity measurements using BacLight bacterial membrane potential kit. The values for WT HG001 in atmospheric were set equal to $100 \%$. Cells were grown to exponential growth phase in atmospheric $(\mathrm{A})$ and $5 \% \mathrm{CO}_{2}\left(\mathrm{CO}_{2}\right)$. As a negative control, the $\mathrm{H}^{+}$ionophore CCCP caused a collapse of the membrane potential in all strains tested both in atmospheric $\mathrm{CO}_{2}$ and in the presence of $5 \% \mathrm{CO}_{2}$. When grown under $5 \% \mathrm{CO}_{2}$ conditions, $\mathrm{HGOO} 1$ and $\triangle m p s A B C$ showed significantly higher relative membrane potential ${ }^{* *} p<0.01$ and ${ }^{\star * \star *} p<0.0001$, respectively, as determined by unpaired two-sided $t$-test) compared with their corresponding strains in atmospheric conditions. Each bar shown in the graph represents the mean value \pm standard error mean (SEM) from four independent experiments (three independent experiments for CCCP), each with three technical replicates. c mpsA expression in three wild-type Staphylococcus aureus strains HG001, USA300, and MW2 under atmospheric and 5\% $\mathrm{CO}_{2}$ conditions. The relative expression of mpsA under $5 \% \mathrm{CO}_{2}$ conditions, normalized to its corresponding strains grown in atmospheric (A) conditions. Only mpsA expression in HG001 is significantly lower in $5 \% \mathrm{CO}_{2}$ compared with atmospheric conditions; ${ }^{*} p<0.05$ by unpaired two-sided $t$-test. (ns = not significant). Each bar shown in the graph represents the mean value \pm standard error mean (SEM) from four independent experiments, each with three technical replicates. Source data are provided as a Source Data file

$m p s A$ expression was downregulated under $5 \% \mathrm{CO}_{2}$ conditions. Since the $m p s$ deletion mutants, except $\Delta m p s C$, are responsive to $5 \% \mathrm{CO}_{2}$, we postulated that the expression of $m p s A B$ could be affected by $\mathrm{CO}_{2}$. To investigate whether high $\mathrm{CO}_{2}$ levels influence mps transcription, Quantitative Reverse Transcriptase-PCR (qRT-PCR) was performed to determine the transcription of $m p s A$ in atmospheric and $5 \% \mathrm{CO}_{2}$ conditions. To exclude strain specific effects, three different WT $S$. aureus strains were used, namely HG001, USA300 LAC, and MW2 (USA400). RNA was isolated during the exponential growth phase and the mRNA of $m p s A$ from the three strains grown in atmospheric air and in $5 \% \mathrm{CO}_{2}$ were compared. Transcript abundance for each strain cultivated in $5 \% \mathrm{CO}_{2}$ was normalized to its corresponding strain grown in atmospheric conditions. Relative to the growth in atmospheric air, mpsA in HG001 grown in $\mathrm{CO}_{2}$ was downregulated the most, namely 2.5 -fold, in USA300 2-fold, and in MW2 1.7-fold (Fig. 3c). In short, mpsA expression was lower in $5 \% \mathrm{CO}_{2}$ compared with atmospheric conditions in all three strains tested; however, only $m p s A$ downregulation in HG001 reached statistical significance $(p<0.05)$. A downregulation of mpsA (SAOUHSC_00412) by $\mathrm{CO}_{2}$ was also observed under cell culture conditions, as reported in Aureowiki ${ }^{19}$.

$\Delta m p s A$ produces fewer hemolytic toxins and is less pathogenic. Because the $\mathrm{CO}_{2}$ /bicarbonate transporter $\mathrm{MpsAB}$ is important for growth and membrane potential generation, we predicted that the mutant would have less toxin production and be less virulent. To examine this question, the hemolytic activity of $\triangle m p s A$ and HG001 grown in atmospheric and $5 \% \mathrm{CO}_{2}$ was tested on sheep blood agar 
a

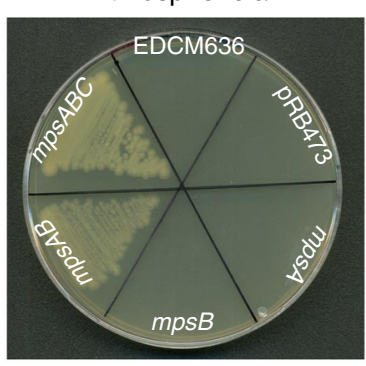

b

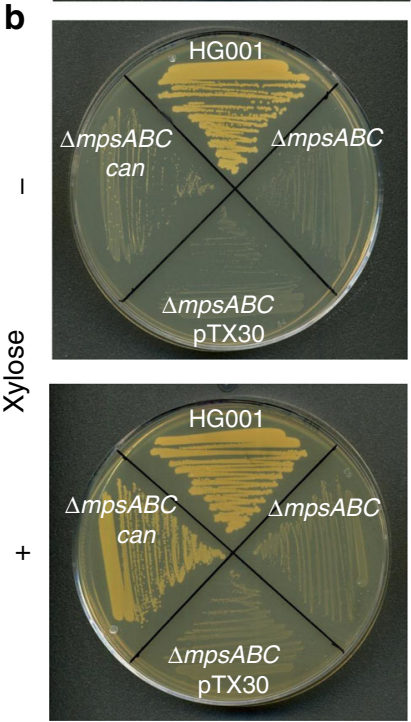

$5 \% \mathrm{CO}_{2}$
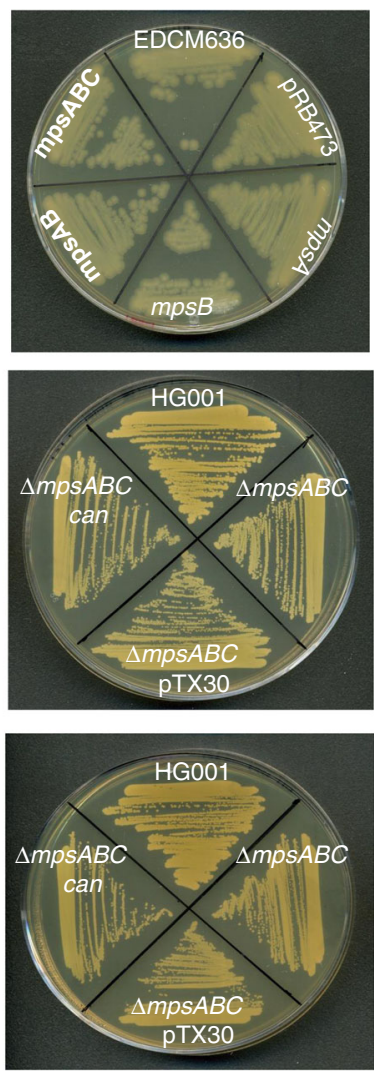

Fig. $4 \mathrm{mps} A B(C)$ complements an $E$. coli carbonic anhydrase mutant and vice versa. a $E$. coli carbonic anhydrase mutant (EDCM636) can be complemented with mpsAB and mpsABC using plasmid pRB473 under the control of its native promoter in atmospheric (left) and $5 \% \mathrm{CO}_{2}$ (right). Clockwise from the top; EDCM636, EDCM636 pRB473 (empty plasmid), EDCM636 pRB473 mpsA, EDCM636 pRB473 mpsB, EDCM636 pRB473 $m p s A B$, and EDCM636 pRB473 mpsABC. b $\triangle m p s A B C$ can be complemented with carbonic anhydrase, can from E. coli MG1655 using pTX30, a xyloseinducible promoter plasmid. Clockwise from the top; wild-type HGO01, $\triangle m p s A B C, \triangle m p s A B C$ pTX30 (empty plasmid), and $\triangle m p s A B C$ pTX30 can. Plates were supplemented with xylose $(+)$ or without xylose $(-)$

followed by cold shock treatment. Consistent with our hypothesis, no visible hemolytic zone was seen in $\triangle m p s A$ when cultivated in atmospheric air, while the presence of $5 \% \mathrm{CO}_{2}$ restored toxin production as indicated by a clear halo on blood agar (Fig. 5a). The same result was also observed for the $\triangle m p s B$ and $\triangle m p s A B C$ mutants (Supplementary Fig. 4). The WT strain HG001 formed a hemolysis zone independent of the presence of $5 \% \mathrm{CO}_{2}$.

Following the finding of decreased toxin production from $\triangle m p s A$, we were interested to find out if this effect is relevant in vivo. To characterize the role of $m p s$ on virulence, Galleria mellonella larvae were used as an invertebrate infection model. The survival rate of larvae injected with WT HG001, $\Delta m p s A$, and PBS as control were observed over the course of 5-day period (Fig. 5b). In the PBS control group, 95\% of the larvae were alive at the end of the 5-day period. In contrast, larvae infected with HG001 showed a survival rate of only $13 \%$ after the 5 -day period, while $53 \%$ of the larvae survived when infected with $\triangle m p s A$. These results indicate that deletion of $m p s A$ rendered $S$. aureus less virulent in an invertebrate infection model.

To assess the contributions of $m p s A B$ to $S$. aureus pathogenesis using an intranasal mouse infection model (pneumonia model), female Balb/c mice were intranasally infected with HG001 and
$\Delta m p s A$. After $48 \mathrm{~h}$, mice were sacrificed and the lungs, kidneys, and livers were recovered to determine the bacterial burden. Although the $\triangle m p s A$ generally exhibited a lower bacterial burden in all three organs, only the bacterial load in the lung was significantly lower compared with its WT strain HG001 (Fig. 5c). These results indicate that the $\Delta m p s A$ is attenuated in pathogenicity compared with its WT strain in this mouse infection model. Taken together, these findings suggest that a functional bicarbonate transport system (mps) is required for fitness and pathogenicity in vivo.

Phylogenetic distribution of $m p s A B$ homologs. Considering the significant effect of $m p s A B$ in $S$. aureus, we examined the phylogenetic distribution of both genes. Searching the RefSeq database revealed genes homologous to $m p s A$ and $m p s B$ were found in a number of species that span the Firmicutes phylum (Fig. 6). These included bacteria from the genus Staphylococcus, Bacillus and a few from Alicyclobacillus, Geobacillus, Parageobacillus, Sulfobacillus, and Thermaerobacter (Fig. 6 and Supplementary Fig. 6). Furthermore, homologs of $m p s A B$ were also widespread in genomes from multiple phyla of prokaryotes (Supplementary Fig. 7). Among those which were well depicted in the phylogenetic tree are from the phyla Proteobacteria, Actinobacteria, and Bacteroidetes. MpsAB are highly conserved in the genus Staphylococcus and to a lesser extent in Bacillus and other Firmicutes (Supplementary Fig. 5). Apart from few exceptions in Actinobacteria and Proteobacteria, in most genomes $m p s A$ and $m p s B$ homologs are always adjacent. In Acidimicrobium ferrooxidans, a ferrous-iron-oxidizing bacterium, mpsA and mpsB homologs exist as a single fused gene (Supplementary Fig. 5).

Apart from $S$. aureus, $m p s A B$ homologs were found in a few human pathogens such as Bacillus anthracis, Legionella pneumophila, and Vibrio cholerae (Table 1). Interestingly, all these three pathogens also carry genes encoding protein with CA domain in addition to $m p s A B$ homologs. In the family of Staphylococcaceae, only Macrococcus caseolyticus JCSC5402, Staphylococcus carnosus TM300, and Staphylococcus pseudintermedius ED99 do not have $m p s A B$ homologs. Instead, they carry genes with CA homologs. This surprising observation also extend to a number of human pathogens; Listeria monocytogenes, Streptococcus pyogenes, Enterococcus faecalis, Enterococcus faecium, Mycobacterium tuberculosis, Helicobacter pylori, Escherichia coli, and Pseudomonas aeruginosa (Table 1). All these important pathogens have CA homologs instead of mpsAB homologs, emphasizing the importance of bicarbonate/ $\mathrm{CO}_{2}$ supply to the microorganisms.

\section{Discussion}

Despite an almost 50-year-old observation of $\mathrm{CO}_{2}$-dependent $S$. aureus growth, little is known about the genetic basis of $S$. aureus regarding its response to changes in $\mathrm{CO}_{2}$ availability. In the present work, we demonstrate that the growth defect of the $\triangle m p s A B C$ mutant under atmospheric $\mathrm{CO}_{2}$ levels could be largely rescued by $5 \%$ $\mathrm{CO}_{2}$ (Fig. 2a) or by $\geq 50 \mathrm{mM}$ bicarbonate (Fig. 2b). Uptake studies with radiolabeled $\mathrm{NaH}^{14} \mathrm{CO}_{3}$ indicates that MpsABC contributed to a significant accumulation of $\mathrm{H}^{14} \mathrm{CO}_{3}{ }^{-}$in the cytoplasm of the WT strain (Fig. 3a). The bicarbonate uptake experiment is tricky because we had to prevent $\mathrm{CO}_{2}$ evaporation as much as possible. This is most likely the reason why we observed increased variations after $5 \mathrm{~min}$. However, one should consider that for substrate uptake, the first 2 min are crucial. During this time range, the standard error mean (SEM) values were really small. We assume that the addition of fluorocitrate was probably not inhibiting the TCA cycle completely; we do not dare to increase the concentration any higher because of the side effects it may cause (we used $10 \mathrm{mM}$ fluorocitrate and the normal concentration used for aconitase enzyme assay is $100 \mu \mathrm{M})$. 
a

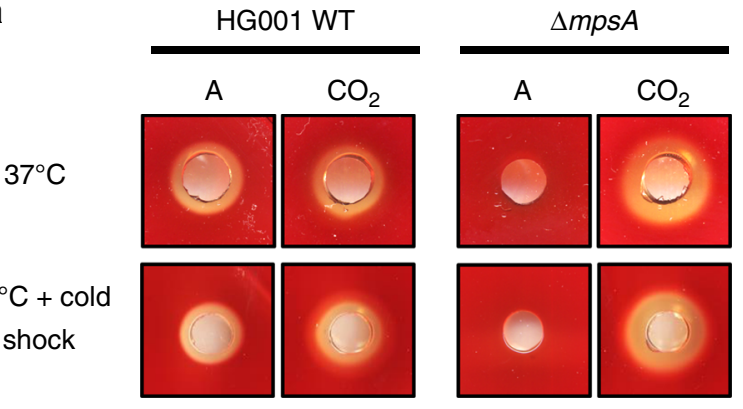

b



C
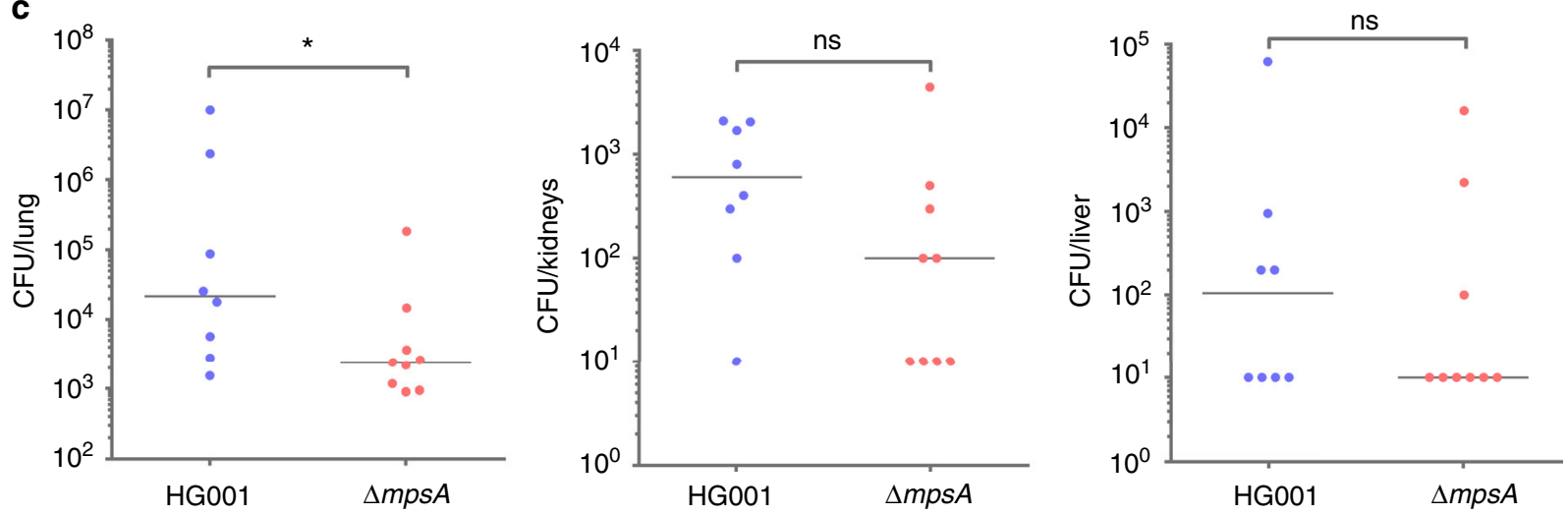

Fig. $5 \Delta m p s A$ produces less hemolytic toxin in atmospheric conditions and is less virulent in animal infection models. a Hemolytic toxin production in $\mathrm{HGO01}$ and $\triangle \mathrm{mps} A$ grown in atmospheric (left) and $5 \% \mathrm{CO}_{2}$ (right) at $37^{\circ} \mathrm{C}$ after overnight incubation (above) and after cold shock treatment (below). b Kaplan-Meier plot of the survival of Galleria mellonella larvae infected with HG001 and $\triangle m p s A$. Larvae injected with PBS were used as control. Larvae infected with $\triangle m p s A$ showed significantly better survival rate compared with the larvae infected with wild-type $H G 001$; ${ }^{\star \star \star \star} p<0.0001$ as determined by Log-rank (Mantel-Cox) test. Results are representative of four independent experiments. c Comparison of wild-type HG001 and $\Delta m p s A$ in a $48 \mathrm{~h}$ intranasal mouse infection model (pneumonia model) in the lung, kidneys, and liver. The bacterial burden of $\Delta m p s A$ in the lung was significantly lower ( $\left.{ }^{\star} p=0.036\right)$ compared with HG001. There was no significant difference (ns) in bacterial burden in the kidney and liver between the two strains. The number of bacteria per organ based on determination of CFU in $n=8$ (WT HG001) and $n=9$ mice ( $\Delta$ mps $A$ ) is shown. The vertical line indicates the median. The detection limit was 10 CFU per organ. Data were analyzed using two-tailed Mann-Whitney test. Source data are provided as a Source Data file

Deletion of the $m p s C$ gene showed no phenotypic alterations or growth defect, suggesting it is not a functional part of the $m p s A B$ operon. Furthermore, the E. coli CA mutant could be complemented with only $m p s A B$, and the gene synteny revealed that $m p s C$ is not collocated with $m p s A B$ homologs in other bacteria than staphylococci. Taken together, these observations speak in favor of MpsAB representing a bicarbonate or DIC transporter with the ability to accumulate intracellular DIC.

CAs are $\mathrm{Zn}^{2+}$ metalloenzymes, which catalyze the reversible hydration of $\mathrm{CO}_{2}\left(\mathrm{CO}_{2}+\mathrm{H}_{2} \mathrm{O} \leftrightarrow \mathrm{HCO}_{3}^{-}+\mathrm{H}^{+}\right)$. For this reason, we hypothesized that a CA mutant in E. coli (EDCM636) could be complemented by mpsAB. The E. coli can gene encodes a $\beta$-class CA, which is essential under atmospheric but not at $5 \%$ $\mathrm{CO}_{2}{ }^{18}$. As expected, the $E$. coli can mutant could be complemented by $m p s A B$ and vice versa; the $S$. aureus $m p s A B C$ mutant could be complemented by E. coli specific can (Fig. 4). Due to the mutual complementation, we thought that MpsB, MpsAB, or MpsABC might have CA activity. Unfortunately, we were unable to detect $C A$ activity using purified proteins or with whole cell extracts. The absence of CA activity in MpsAB suggests that MpsAB and CA have different enzymatic mechanisms, yet they serve the same function; namely, preventing evaporation of 


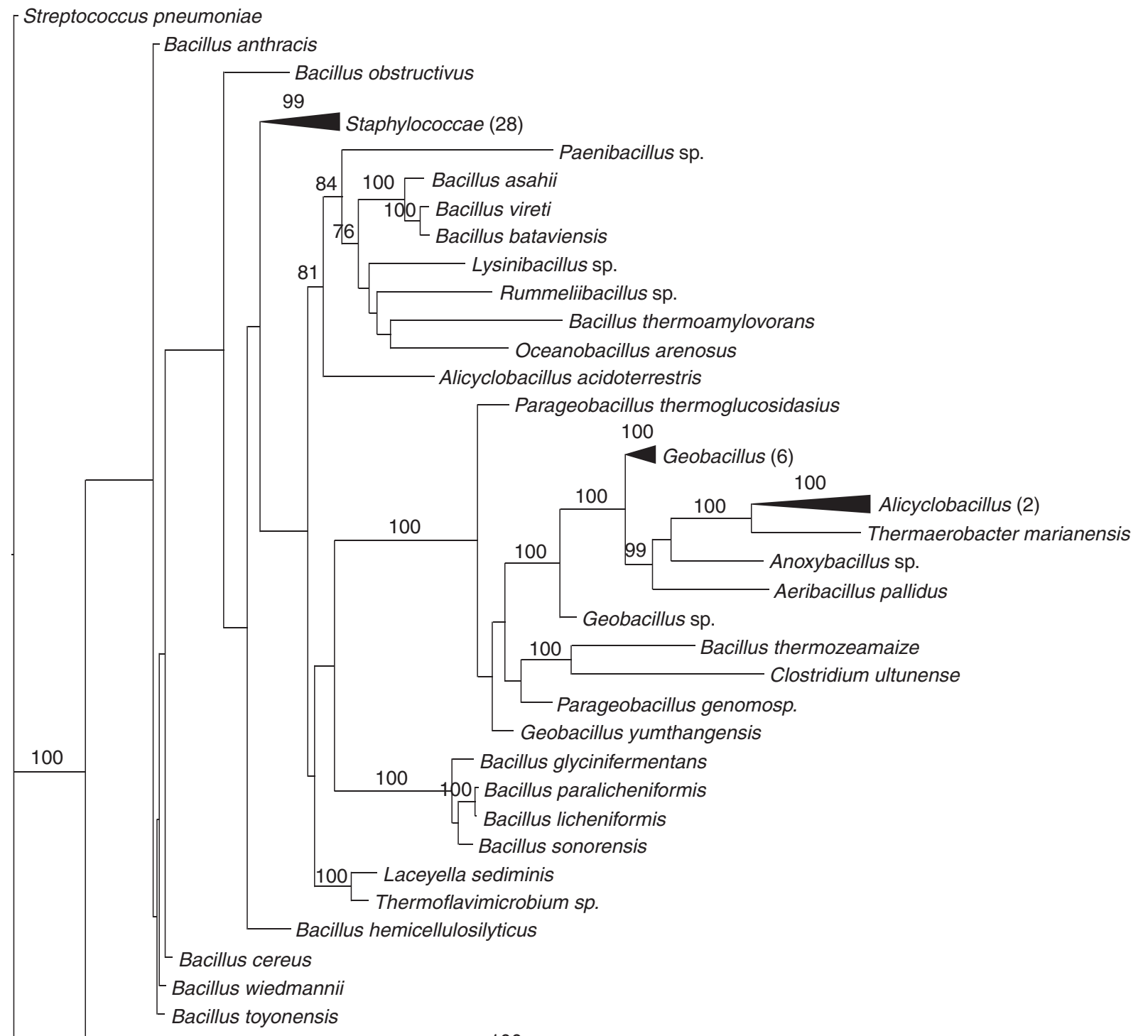

100

Sulfobacillus (5)

Bacillus thuringiensis

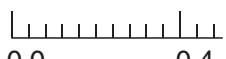

$0.0 \quad 0.4$

Fig. $6 \mathrm{mps} A B$ homologs are widespread in the Firmicutes phylum. Maximum likelihood phylogenetic analysis of concatenated alignments of $m p s A$ and $m p s B$ homologs amongst the phylum Firmicutes. Node support is indicated by bootstrap values from 100 resamplings of the alignment when they exceeded $70 \%$. Collapsed clades are labeled by the genus of associated taxa. The number of taxa belonging to the respective clade is indicated in brackets. Source data are provided as a Source Data file

$\mathrm{CO}_{2}$ by trapping it as bicarbonate for anaplerotic metabolism. Although a considerable amount of $\mathrm{CO}_{2}$ is produced by a functional TCA cycle, this $\mathrm{CO}_{2}$ will be lost in the cytoplasm due to an insufficient concentration for anaplerotic reactions. To prevent this from happening, CA catalyzed the formation of bicarbonate from $\mathrm{CO}_{2}$, which significantly delays the release of intracellular $\mathrm{CO}_{2}$ and providing bicarbonate required for carboxylation reactions $^{20}$.

The observation that EDCM636 could only be complemented by both MpsAB suggests they function together as a transporter. This is also supported by the collocation of mpsAB homologs in many microorganisms across multiple phyla (Fig. 6, Supplementary Figs. 5 and 7). Furthermore, MpsA belongs to PFam00361 ${ }^{21}$, which consists of a diverse family of proton-conducting membrane transporters, including NADH:quinone oxidoreductase subunits (complex I) ${ }^{22}$. In this regard, we demonstrated that MpsABC constitutes a cationtranslocating system, capable of $\mathrm{Na}^{+}$transport similar to the observation regarding $\mathrm{NuoL}^{12}$. MpsB on the other hand belongs to PFam10070, a family of conserved proteins in bacteria of unknown function. Based on these observations, we propose a model that MpsAB is a sodium bicarbonate cotransporter (NBC) as illustrated in Fig. 7. Such NBC transporters are prevalent in mammals ${ }^{23}$, having important functions in maintaining intracellular and whole-body $\mathrm{pH}$, as well as contributing to the transepithelial transport processes. The bicarbonate cotransporters are composed of 13 transmembrane domains $^{24}$, similar to MpsA which has 14 . It is unknown whether mammals also require an MpsB homolog; although the NBC stoichiometry in Xenopus oocytes is $1 \mathrm{Na}^{+}: 2 \mathrm{HCO}_{3}{ }^{-}$, indicating a 
Table 1 Distribution of MpsAB and carbonic anhydrase (CA) homologs in various species

\section{Species}

MpsAB homolog CA homolog

Staphylococcus aureus aureus

NCTC 8325

Staphylococcus carnosus

carnosus TM300

Staphylococcus pseudintermedius ED99

Macrococcus caseolyticus JCSC5402

Bacillus anthracis delta Sterne

Bacillus subtilis subtilis 168

Listeria monocytogenes $R 479 a$

Streptococcus pneumoniae R6

Streptococcus pyogenes A20

Enterococcus faecalis V583

Enterococcus faecium DO

Mycobacterium tuberculosis H37Rv

Helicobacter pylori 199

Escherichia coli 0157:H7 Sakai (EHEC)

Legionella pneumophila pneumophila

Philadelphia-1

Pseudomonas aeruginosa PAO1

Vibrio cholerae sv. $\mathrm{O} 1 \mathrm{bv}$. El

Tor N16961

Homologies were inferred based on PFam domains search from finished bacterial genomes in Integrated Microbial Genomes \& Microbiomes (IGM/G) database. MpsA, MpsB, prokaryotic type-CA, and eukaryotic-type CA belongs to PFam00361, PFam10070, PFam00484, and PFam00194, respectively. The symbol $+/$ - indicates the presence or absence of homolog, while (a) and (b) indicates prokaryotic and eukaryotic-CA, respectively. Asterisk indicates that one of the three CAs is a gene fusion and has a probable transmembrane domain in which the $\mathrm{N}$-terminal part has PFam00916 (sulfate transporter family) and PFam00484 in the C$\mathrm{N}$-terminal part
terminal part

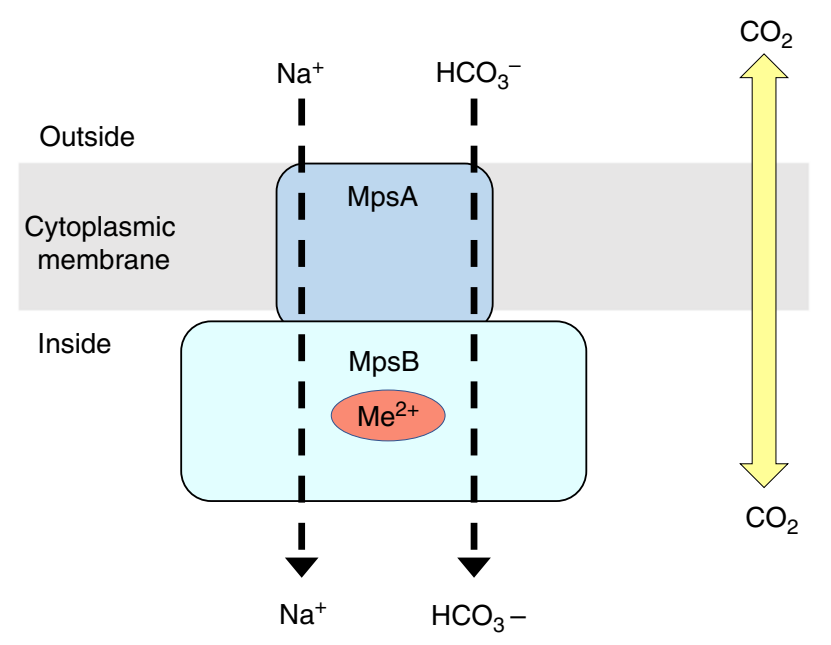

Fig. 7 Proposed function for MpsAB. We proposed that MpsAB represents a sodium bicarbonate cotransporter (NBC). These transporters are prevalent in mammalians and are expressed throughout the body. Unlike $\mathrm{CO}_{2}$ which can diffuse passively in and out of the cells, bicarbonate $\left(\mathrm{HCO}_{3}{ }^{-}\right)$needs to be transferred into the cells via a transporter. In S. aureus, MpsAB most likely function together as cotransporter to transport $\mathrm{Na}^{+}$and $\mathrm{HCO}_{3}-. \mathrm{Me}^{2+}$ indicates a metal binding site in $\mathrm{MpsB}$

possible accessory protein interaction ${ }^{25}$. As $\mathrm{CO}_{2}$ is volatile, transporting bicarbonate or DIC helps to "trap" it in the cytoplasm for the carboxylation reactions of anaplerotic metabolism. In Firmicutes, these include pyruvate carboxylase fuelling the TCA cycle, acetylCoA-carboxylase as the first enzyme of fatty acid synthesis, or phosphoribosylaminoimidazole carboxylase involved in purine biosynthesis.

Phylogenetic analysis revealed that $m p s A B$ are widespread, not only among Firmicutes, but also across multiple phyla (Fig. 6 and Supplementary Fig. 7). Bacteria that possess homologs of MpsAB have diverse lifestyles; for example hyperthermophilic (e.g., Aquifex aeolicus), thermophilic and acidophilic (e.g., Acidimicrobium ferrooxidans and Acidithiobacillus caldus), $\mathrm{CO}_{2}$ fixing bacteria (e.g., Chloroflexus aurantiacus), and nitrogen-fixing bacteria (e.g., Bradyrhizobium oligotrophicum and Frankia sp). In addition, MpsAB homologs were also described in the gammaproteobacterial chemolithoautotroph Thiomicrospira crunogena ${ }^{15}$. Similar to $S$. aureus, the T. crunogena genes Tcr_0853 (mpsA homolog) and Tcr_0854 (mpsB homolog) were cotranscribed, constitute a probable DIC transporter, and its mutants required elevated DIC for growth ${ }^{15}$. Although T. crunogena has $\mathrm{CO}_{2}$ concentrating mechanisms (CCMs), its DIC uptake process is unclear due to the lack of cyanobacterial transporter homologs. That being said, it is assumed that in autotrophic bacteria, a DIC concentrating system is necessary to ensure that Rubisco is saturated with $\mathrm{CO}_{2}$, so that carboxylation proceeds at its maximum rate.

Another problem with the "ancient" Rubisco is that it also reacts with oxygen ${ }^{26}$. In this respect, the atmospheric $\mathrm{CO}_{2}$ content in ancient times was high, while that of $\mathrm{O}_{2}$ was low. In a $\mathrm{CO}_{2}$-rich environment, the $\mathrm{CO}_{2}$-fixing enzymes in autotrophic bacteria, such as Rubisco could work properly despite its low affinity and limited discrimination between $\mathrm{CO}_{2}$ and $\mathrm{O}_{2}$. However, due to oxygenic photosynthesis in the subsequent 2.5 billion years, the atmosphere became $\mathrm{O}_{2}$ rich and $\mathrm{CO}_{2}$ poor ${ }^{27}$. As a consequence, the cytoplasmic $\mathrm{CO}_{2}$ levels became too low for proper $\mathrm{CO}_{2}$ fixation and assimilation ${ }^{28,29}$. Therefore, it is assumed that autotrophic bacteria that grow in atmosphere appear to uniformly have $\mathrm{CCMs}^{27}$. Bacterial CCM involves two main functions: (a) active transport of inorganic carbon species $\left(\mathrm{HCO}_{3}{ }^{-}\right.$and $\left.\mathrm{CO}_{2}\right)$ to concentrate $\mathrm{HCO}_{3}{ }^{-}$within the cell, and (b) the pool of $\mathrm{HCO}_{3}{ }^{-}$is then utilized to provide elevated $\mathrm{CO}_{2}$ concentrations around primary $\mathrm{CO}_{2}$ fixing enzyme, Rubisco, encapsulated in carboxysomes which also contain $\mathrm{CA}^{14}$. Recently, DIC transporters from four evolutionary distinct families were described in several genera of sulfur-oxidizing chemolithoautotrophs ${ }^{16}$. Two of these DIC transporters demonstrate DIC uptake even though they share low protein identity with the wellcharacterized cyanobacterial transporters, SbtA- and SuIP-family bicarbonate transporters. SbtA is an inducible, high-affinity $\mathrm{Na}^{+}$-dependent $\mathrm{HCO}_{3}{ }^{-}$transporter ${ }^{30}$, while BicA, a member of the SuIP family, is a low-affinity, high-flux $\mathrm{Na}^{+}$-dependent $\mathrm{HCO}_{3}{ }^{-}$transporter ${ }^{31}$. Heterologously expressed chromate ion transporter from Hydrogenovibrio thermophilus JR2 is also capable of DIC uptake ${ }^{16}$. The fourth type of transporter is a two component DIC transporter encoded by the genes Tcr_0853 and Tcr_0854 in Hydrogenovibrio crunogenus (previously known as $T$. crunogena) as discussed previously ${ }^{15,16}$. Another wellcharacterized DIC transporter expressed in cyanobacteria is BCTI, encoded by the $c m p A B C D$ operon is an inducible, highaffinity $\mathrm{HCO}_{3}{ }^{-}$transporter belonging to the ATP binding cassette transporter family ${ }^{32}$. It is thought that these transporters differ in their affinities and mechanisms for transport (as cation symporter or anion antiporter), which provide advantages under specific growth conditions ${ }^{16}$. Out of all these distinct transporters families, MpsAB belongs to the same type as encoded by Tcr_0853 and Tcr_0854 from H. crunogenus in terms of function and homology; MpsAB is $29-30 \%$ identical to Tcr_0853 and Tcr_0854. Such transporters and their function were so far not described in Firmicutes.

Staphylococci, like most other Firmicutes, are neither autotrophic nor do they possess typical carboxysome structures or Rubisco. The question is, therefore, why do they need MpsAB as a DIC concentrating system. The most rational answer would be that enzymes involved in substrate carboxylation are not sufficiently supplied with $\mathrm{HCO}_{3}^{-}$under atmospheric $\mathrm{CO}_{2}$, thus 
require a higher $\mathrm{HCO}_{3}{ }^{-}$concentration for proper activity. For example, the biotin-dependent pyruvate carboxylase has a central function in fueling the TCA cycle with oxaloacetate. If this reaction is impaired, not only the TCA cycle but also respiration and the membrane potential would be impaired. This is exactly the observed phenotype of the $m p s A B C$ mutant, which exhibited the typical features of SCV in atmospheric $\mathrm{CO}_{2}$ and similar to the S. aureus hemB mutant ${ }^{10,12}$. It is also tempting to speculate that MpsAB serve to prevent the depletion of cellular DIC, a role which is conventionally played by CA or other known cyanobacterial bicarbonate transporter. S. aureus has neither of them, however since $\mathrm{CO}_{2}$ or $\mathrm{HCO}^{-}$is crucial for many cellular processes, a DIC concentrating mechanism is likely to be important for its growth and survival. As a major pathogen S. aureus needs to adapt to different niches; as commensal inhabitant on environmentally exposed skin and mucosal surfaces to colonization and infection inside the mammalian host. Variations of $\mathrm{CO}_{2}$ levels are one of the evident conditions it would encounter in this process, and having a DIC concentrating mechanism would ensure its sufficient supply intracellularly. Collectively, these hypotheses could explain the role of MpsAB beyond the known carbon-fixing bacteria.

Due to the interchangeable role of $\mathrm{MpsAB}$ and $\mathrm{CA}$, we sought to compare the occurrence of both the genes in Firmicutes. In this phyla, there is tendency that either one of them is present; in some cases both genes are present in one strain/species, for example in B. anthracis and B. subtilis (Table 1). This same observation appears to hold true for selected species of Grampositive and Gram-negative pathogens (Table 1). Interestingly, highly pathogenic species for example Legionella pneumophila and Vibrio cholera have MpsAB homologs as well as at least two CA homologs, while Mycobacterium tuberculosis, Helicobacter pylori, E. coli O157:H7, and Pseudomonas aeruginosa have two or even three CAs. The importance of the distribution of MpsAB and $\mathrm{CA}$ in the respective strains or species is not yet clear. Nevertheless, the occurrence of two and even three CA genes, and/or MpsAB suggests that DIC concentrating mechanism play an important role in fitness and pathogenicity. We assume that the decreased virulence of the $m p s A$ mutant is due to the very low intracellular bicarbonate levels. Bicarbonate is the substrate for biotin carboxylase that produces carboxybiotin, the substrate for other carboxylases, such as PEP-, or pyruvate carboxylase that feed the TCA cycle. For example, the $K_{\mathrm{m}}$ value for bicarbonate of the $E$. coli biotin carboxylase is comparatively high at $16 \mathrm{mM}^{33}$, indicating that the enzyme has a very low affinity for bicarbonate. This suggests that a high level of intracellular bicarbonate is needed to fuel the TCA cycle, respiration, fatty acid biosynthesis, and membrane potential. ATP and membrane potential are important for toxin secretion.

The virulence attenuation of the $\triangle m p s A$ was also seen in vivo. In the Galleria mellonella larvae survival model, the killing rate by the mutant was significantly decreased (Fig. 5b). In the intranasal S. aureus mouse infection model (pneumonia model), the CFU/ lung was also significantly decreased with $\triangle m p s A$ (Fig. 5c). We have chosen a lung infection model because the $\mathrm{CO}_{2}$ content in the lung is higher and we expect that the $m p s A$ mutant might have a better chance to grow. $\mathrm{CO}_{2}$ is produced during respiration and is carried by the blood through the venous system to the lungs, where it is exhaled with a concentration of $\sim 3.8 \%$ in the tidal air or 4501 of $\mathrm{CO}_{2}$ per day ${ }^{34}$. There was no statistically significant difference in bacterial burden in the kidney and liver between HG001 and the mpsA mutant. This can be explained that the lung was the primary organ that was infected with S. aureus. From there, S. aureus spreads to other organs during the course of infection. Spreading is a complex process as the bacteria have several barriers to pass: first the lung epithelium and endothelium of blood vessels, and then again the endothelium of blood vessels to establish infections in other organs. Thus, infection burden in kidneys and liver was lower than in the lung, as it has also been observed by Lee et al. ${ }^{35}$.

In conclusion, we propose that MpsAB constitutes a DIC transporter that is important for growth of $S$. aureus under low, atmospheric $\mathrm{CO}_{2}$ conditions. This transporter system underlies the importance of $\mathrm{CO}_{2} /$ bicarbonate in the fitness and pathogenicity of S. aureus and may help shed some light on earlier observations about $\mathrm{CO}_{2}$-dependent variants. Furthermore, $m p s A B$ homologs are widespread in diverse groups of microorganisms that adopt different lifestyles and occupy a wide range of niches. In contrast to the dogma of DIC transporters, MpsAB plays an important function even in non-autotrophic bacteria by concentrating bicarbonate for anaplerotic pathways that are relevant across most microorganisms. An interesting finding in our study was that MpsAB and CA could functionally replace one another in our mutual complementation experiments, further supporting the essentiality of bicarbonate. Within this context, a number of the pathogenic species have either MpsAB or CA, whereas both are present in some of these species, suggesting their roles in growth and virulence.

\section{Methods}

Bacterial strains and growth conditions. The strains used in this study are listed in Supplementary Table 1 . For cloning procedures, all staphylococcal and E. coli strains were cultivated at $37^{\circ} \mathrm{C}$ with shaking at $150 \mathrm{rpm}$ in basic medium (BM) (1\% soy peptone, $0.5 \%$ yeast extract, $0.5 \% \mathrm{NaCl}, 0.1 \%$ glucose, and $0.1 \% \mathrm{~K}_{2} \mathrm{HPO}_{4}, \mathrm{pH} 7.2$ ), unless specified otherwise. All cultures were grown in $10 \mathrm{ml}$ medium using baffled $100 \mathrm{ml}$ shake flasks (flask-to-medium ratio 10:1) as recommended ${ }^{36}$,

with the exception of growth studies, which were grown in $15 \mathrm{ml}$ medium (flask-tomedium ratio $7: 1$ ). The medium was supplemented with the following antibiotics, where applicable at the indicated final concentrations: chloramphenicol at $10 \mu \mathrm{g} \mathrm{ml}^{-1}$ tetracycline at $25 \mu \mathrm{g} \mathrm{ml}^{-1}$, for staphylococcal strains and $100 \mu \mathrm{g} \mathrm{ml}^{-1}$ ampicillin and $30 \mu \mathrm{g} \mathrm{ml}^{-1}$ kanamycin for E. coli strains.

Growth studies of staphylococcal strains. TSB (Sigma-Aldrich) and TSA plates (Sigma-Aldrich) were used for growth studies involving S. aureus. For growth characterization on solid medium, $S$. aureus strains were precultured for $24 \mathrm{~h}$ at $37^{\circ} \mathrm{C}$ under continuous shaking starting from a single colony. Cultures were then diluted to $10^{-5}-10^{-7}$ and an aliquot of $100 \mu$ l was plated on TSA plates. The plates were incubated for 5 days at $37^{\circ} \mathrm{C}$ under atmospheric and $5 \% \mathrm{CO}_{2}$ conditions. The $5 \% \mathrm{CO}_{2}$ level was achieved in a $\mathrm{CO}_{2}$ incubator (Heraeus Instruments). The colony size and appearance on TSA was observed every $24 \mathrm{~h}$ for 5 days and documented with a Leica M125 stereomicroscope. For growth characterization in liquid medium, $S$. aureus cells were precultured in TSB as described above. S. aureus strains involved were WT HG001, $\triangle m p s A, \triangle m p s B, \triangle m p s C$, and $\triangle m p s A B C$ that were transformed with pRB473-mpsA, pRB473-mpsB, pRB473-mpsC, and pRB473$m p s A B C$, respectively. Main cultures were inoculated to $\mathrm{OD}_{578}=0.05$ and grown under atmospheric and $5 \% \mathrm{CO}_{2}$ conditions at $37^{\circ} \mathrm{C}$ under continuous shaking. Aliquots were taken at $2,4,6,8,10,12,24,48$, and $72 \mathrm{~h}$ for $\mathrm{OD}_{578}$ measurements.

Growth studies of $\triangle m p s A B C$ with bicarbonate supplemented media. Media were supplemented with $\mathrm{NaHCO}_{3}$. Precultures were grown as described above. Main cultures were cultivated as described above as well, but with the addition of the supplements at final concentrations of $1,5,10$, and $50 \mathrm{mM}$ and grown under atmospheric conditions. $\triangle m p s A B C$ grown under atmospheric and $5 \% \mathrm{CO}_{2}$ conditions were used as controls.

Complementation experiments of $\triangle m p s A B C$ and EDCM636. With regards to $S$ aureus $\triangle m p s A B C$ complemented with can from $E$. coli, the cultures were inoculated and were adjusted to $\mathrm{OD}_{578}=5$, then the strains were streaked on agar plates containing no xylose or $0.5 \%$ xylose (filter sterilized). Complementation of EDCM636 with pRB473-mpsA, pRB473-mpsB, pRB473-mpsAB, and pRB473$m p s A B C$, were also performed as described above, with the exception that BM and BMA were used instead of TSB and TSA.

Construction of the $\boldsymbol{S}$. aureus deletion mutants. All oligonucleotides used in this study are listed in Supplementary Table 2. Nucleotide and amino acid sequences were obtained from the Kyoto Encyclopedia of Genes and Genomes (KEGG). The deletion mutants $S$. aureus HG001 $\triangle m p s B$ (KEGG accession no.

SAOUHSC_00413) and $\triangle m p s C$ (SAOUHSC_00414) were constructed as markerless deletions using allelic replacements as described by Bae and Schneewind ${ }^{37}$. 
Briefly, $1 \mathrm{~kb}$ upstream and $1 \mathrm{~kb}$ downstream of $m p s B$ and $m p s C$, respectively were amplified from the chromosomal DNA of S. aureus HG001. The fragments were assembled with linearized (Bg1II restriction enzyme) plasmid pBASE $6^{38}$ by Gibson assembly ${ }^{39}$ using Hi-Fi DNA Assembly Master Mix (New England Biolabs). The resulting plasmids were first introduced into $E$. coli $\mathrm{DC} 10 \mathrm{~B}^{40}$ and then into $S$. aureus RN4220 via electroporation before transformation of S. aureus HG001. Deletion of the genes were confirmed by PCR and sequence analysis. For $S$. aureus HG001 $\triangle m p s A$ and $\triangle m p s A B C$, pKOR1 ${ }^{37}$ carrying up- and downstream flanking regions of $\sim 2 \mathrm{~kb}$ each for both deletions ${ }^{12}$ were used.

\section{Construction of complementation vectors. Complementation of $\triangle m p s B$ was} carried out with plasmid pCtufamp ${ }^{41}$ and $\triangle m p s C$ with plasmid $\mathrm{pRB} 473^{42}$. For construction of complementation vector for $m p s B, m p s B$ was amplified and then assembled into linearized pCtufamp plasmid (HindIII and PacI restriction enzyme) by Gibson assembly. As for construction of complementation vector for $m p s C$, the putative native promoter sequence for $m p s A B C$ as well as $m p s C$ were amplified and then inserted into linearized pRB473 (EcoRI restriction enzyme). The putative promoter regions were determined by DNA sequence analysis. The constructed plasmids were first introduced into E. coli DC10B then into S. aureus RN4220 before being transformed into S. aureus HG001 $\triangle m p s B$ and $\triangle m p s C$, respectively. For complementation of $\triangle m p s A$ and $\triangle m p s A B C$, recombinant vector plasmids pRB473-mpsA and pRB473-mpsABC from previous study ${ }^{12}$ were isolated and the corresponding deletion mutants were transformed as described above. In addition, all constructed pRB473 derivatives were transformed into EDCM636, an E. coli MG1655 derivative harboring a kanamycin resistance marker replacing a deletion of the CA encoding gene can. It was purchased from E. coli Genetic Stock Center, Yale University. Additional complementation vectors pRB473-mpsB and $\mathrm{pRB} 473$ $m p s A B$ were constructed and transformed into EDCM636, including the empty vector.

Construction of pTX30-can. The xylose inducible plasmid pTX30 $0^{43}$ was used for expression of can (KEGG accession no. b0126). Therefore, can was amplified from the chromosomal DNA of E. coli MG1655. The insert was inserted into the linearized pTX30 (BamH1 and EcoR1 restriction enzymes) using T4 DNA ligase (Thermo Scientific). The resulting plasmids was transformed into $S$. aureus RN4220 via electroporation before being transformed into $\triangle m p s A B C$.

Quantitative RT-PCR. qRT-PCR was carried out to determine the gene expression of $m p s A$ in three WT S. aureus strains, namely HG001, USA $300^{44}$, and MW2 $2^{45}$ under atmospheric and $5 \% \mathrm{CO}_{2}$ conditions. All bacteria precultures were precultivated in atmospheric and $5 \% \mathrm{CO}_{2}$ conditions, respectively, for $24 \mathrm{~h}$. Main cultures were inoculated at an $\mathrm{OD}_{578}=0.1$ for each strain and grown in its respective growth conditions for $3 \mathrm{hrs}$. Subsequently an aliquots of $\mathrm{OD}_{578}=2.0$ were harvested. RNA isolation was carried out using a RNeasy Mini kit (Qiagen) according to manufacturer's instructions. The resulting RNA was treated with RQ1 RNase-Free DNase (Promega). Subsequently, the RNA was analyzed for DNA contamination using ReproFast DNA Polymerase (Genaxxon Bioscience) with noRT control PCR. Power SYBR ${ }^{\circledR}$ Green RNA-to-CT ${ }^{\text {Tx }} 1$ 1-Step Kit (Applied Biosystems) was used in AviaMx Real-Time PCR System (Agilent Technologies) with primers directed to $m p s A$ using $50 \mathrm{ng}$ of RNA as template in each reaction. DNA gyrase subunit B (gyrB) was used as housekeeping gene control. The RNA used in qRT-PCR was isolated from four independent experiments. The relative expression of $m p s A$ under $5 \% \mathrm{CO}_{2}$ was normalized to its corresponding strains grown in atmospheric conditions.

Bicarbonate uptake analysis. Main cultures were inoculated to an $\mathrm{OD}_{578}=0.1$ and grown until exponential growth phase for $3 \mathrm{~h}$ (S. aureus HG001) and $18 \mathrm{~h}$ $(\triangle m p s A B C)$. Cells were then centrifuged and washed with $10 \mathrm{mM}$ Tris buffer at $\mathrm{pH}$ 7. Cells were resuspended in Tris buffer and adjusted to $\mathrm{OD}_{578}$ of 1 in a final volume of $10 \mathrm{ml}$. Fluorocitrate and glucose at a final concentration of $10 \mathrm{mM}$ and $5 \mathrm{mM}$, respectively, were added to both cell suspensions before incubated at room temperature with magnetic stirring for $30 \mathrm{~min}$. After that, $50 \mu \mathrm{Ci}$ of $\mathrm{NaH}^{14} \mathrm{CO}_{3}$ (specific activity $58 \mathrm{mCi} \mathrm{mmol}^{-1}$, Hartmann Analytic) was added. One milliliter of samples was collected at time 0 (before the addition of $\mathrm{NaH}^{14} \mathrm{CO}_{3}$ ), $0.5,1,2,4,6,8$, 10 , and $15 \mathrm{~min}$. After each sample was collected, cells were immediately filtered by vacuum filtration onto membrane filters (Whatman ${ }^{\mathrm{TM}} \mathrm{ME} 25$ mixed cellulose ester membrane filters $0.45 \mu \mathrm{m}$ ) and washed with $10 \mathrm{ml}$ of Tris $\mathrm{NaCl}$ buffer $(10 \mathrm{mM}$ Tris with $100 \mathrm{mM} \mathrm{NaCl}$ ). The membrane filters were then placed in a vial containing $10 \mathrm{ml}$ of liquid scintillation cocktail (Ultima Gold, PerkinElmer). Radioactivity retained on the membrane filters was taken as $\mathrm{H}^{14} \mathrm{CO}_{3}$ uptake which was determined by ${ }^{14} \mathrm{C}$ accumulation in cells, measured by liquid scintillation counting (LKB Wallac 1209 RackBeta liquid scintillation counter). Results were recorded as counts per minute (CPM).

Hemolysis assay. To analyze hemolytic toxin production, overnight cultures of $\mathrm{HG} 001 \triangle m p s A, \triangle m p s B$, and $\triangle m p s A B C$ grown in $5 \% \mathrm{CO}_{2}$ were used. Each bacterial strain was adjusted to an $\mathrm{OD}_{578}$ of 2 and streaked onto blood agar (Oxoid). In addition, to better visualize the hemolysin halo zones, cultures were grown in atmospheric (16 h for HG001 and $48 \mathrm{~h}$ for $\triangle m p s A$ ) and in $5 \% \mathrm{CO}_{2}$ conditions $(16 \mathrm{~h}$ for both HG001 and $\triangle m p s A$ ). An aliquot of same $\mathrm{OD}_{578}$ was collected for each strain before being centrifuged to remove the cell pellets. The remaining supernatant was filtered and concentrated with SpeedVac. The concentrated supernatant was then dropped into blood agar. All plates were incubated at $37^{\circ} \mathrm{C}$ under atmospheric and $5 \% \mathrm{CO}_{2}$ conditions respectively for $24 \mathrm{~h}$ before subjected to cold shock treatment for $72 \mathrm{~h}$.

Galleria mellonella larvae infection model. Final-stage instar larvae of G. mellonella were purchased from Reptilienkosmos.de, Germany. Ten larvae weighing between 300 and $600 \mathrm{mg}$ were infected with S. aureus HG001 and $\triangle m p s A$, respectively. Bacteria were grown in TSB at $37^{\circ} \mathrm{C}$ with shaking for $24 \mathrm{~h}$ (HG001) and $48 \mathrm{~h}(\triangle m p s A)$. Cells were washed twice and resuspended in Dulbecco's Phosphate-Buffered Saline (DPBS) (Gibco ${ }^{\mathrm{TM}}$ ) before being adjusted to an $\mathrm{OD}_{578}$, which corresponds to $5 \times 10^{8}$ colony-forming unit (CFU) for each strain. Each larva was injected with $5 \times 10^{6}$ cells. Larvae injected with DPBS served as control group. The larvae were incubated at $37^{\circ} \mathrm{C}$ for 5 days after infection and surviving larvae were counted every day starting from $24 \mathrm{~h}$ after infection. The experiments were replicated four times. Data collected from each independent experiment were pooled for statistical analysis, resulting in $n=40$ for every strain.

Membrane potential measurement. Main cultures were grown to exponential growth phase for $\mathrm{HG001}$ ( $3 \mathrm{~h}$ in atmospheric and $5 \% \mathrm{CO}_{2}$, respectively) and also for $\triangle m p s A B C$ ( $18 \mathrm{~h}$ in atmospheric and $3 \mathrm{~h}$ in $5 \% \mathrm{CO}_{2}$ conditions). Cells were washed once in phosphate-buffered saline (PBS) before adjusted to an $\mathrm{OD}_{578}$ of 0.4 . Membrane potential was determined using BacLight bacterial membrane potential kit (Invitrogen) according to the manufacturer's protocol. Briefly, $10 \mu \mathrm{l}$ of the dye 3,3'-diethyloxacarbocyanine iodide (DiOC2[3]) was added to the bacterial suspension samples and incubated for $30 \mathrm{~min}$. Then, $200 \mu \mathrm{l}$ of the stained or unstained sample was applied to a black, flat-bottomed 96-well plate to determine the red and green fluorescence intensity using a microplate reader (Tecan Infinite M200). Excitation and emission wavelengths were chosen as recommended by the kit's protocol. The fluorescence intensities of wild-type HG001 grown in atmospheric was set equal to $100 \%$.

Mouse infection experiments. Overnight cultures of HG001 and $\triangle m p s A$ in Brain Heart Infusion Broth (BHI) medium were diluted to a final $\mathrm{OD}_{600}$ of 0.05 in $50 \mathrm{~m}$ fresh BHI medium and grown until exponential growth phase (3.5 h for HG001 and $6 \mathrm{~h}$ for $\triangle m p s A$ ) at $37^{\circ} \mathrm{C}$ with $5 \% \mathrm{CO}_{2}$. After centrifugation, the cell pellet was resuspended in $\mathrm{BHI}$ with $20 \%$ glycerol, aliquoted and stored at $-80^{\circ} \mathrm{C}$. For the generation of in vivo infection, aliquots were thawed and washed twice with PBS. The infectious dose used for infection was very similar for both strains $\left(2.1 \times 10^{8}\right.$ $\mathrm{CFU} / \mathrm{HG} 001$ and $1.7 \times 10 \mathrm{CFU} / \triangle m p s A$ in $20 \mu \mathrm{l}$ ). A sample of the infection inoculum was plated on TSB agar plates in order to control the infection dose. For the intranasal S. aureus infection model (pneumonia model) we used female Balb/c mice (8 or 9 per group, 6 weeks, Janvier Labs, Le Genest-Saint-Isle, France). They were intranasally infected with $2.1 \times 10^{8} \mathrm{CFU}$ of HG001 and with $1.7 \times 10^{8} \mathrm{CFU}$ of $\triangle m p s A$. During infection, mice were scored twice a day and the severity of infection was determined accordingly to recognize if humane endpoint was reached. After $48 \mathrm{~h}$ of infection, mice were sacrificed, the lungs, kidneys, and livers recovered, homogenized and plated in serial dilutions on TSB agar plates incubated overnight at $37^{\circ} \mathrm{C}$ with $5 \% \mathrm{CO}_{2}$ in order to determine the bacterial burden. Significant difference in the CFU counts in the organs between the two groups was determined with Mann-Whitney test.

Ethics statement. All of the animal studies were approved by the local government of Lower Franconia, Germany (approval number 55.2-2532-2-155) and performed in strict accordance with the guidelines for animal care and experimentation of German Animal Protection Law and the DIRECTIVE 2010/63/EU of the EU. The mice were housed in individually ventilated cages under normal diet in groups of four to five throughout the experiment with ad libitum access to food and water.

Phylogenetic analysis of $\boldsymbol{m p s} \boldsymbol{A}$ and $\boldsymbol{m p s} \boldsymbol{B}$ homologs. Homologs of $m p s A$ (YP_498998.1) and mpsB (YP_498999.1) from S. aureus were identified from the RefSeq database ${ }^{46}$ using Microbial Protein BLAST. All searches were either conducted within the whole Bacteria domain, or restricted to the bacterial phylum Firmicutes or the bacterial genus Staphylococcus. Since several species have more than one putative homologs of $m p s A$ and $m p s B$, we restricted the hits only to those protein pairs of $m p s A$ and $m p s B$, whose coding sequences, extracted from the respective GenBank files, are in immediate proximity in the genome. The protein sequences of $m p s A$ and $m p s B$ homologs were aligned separately using Clustal Omega (version 1.2.1) ${ }^{47}$. The multiple sequence alignments (MSAs) were subsequently concatenated into one single MSA comprising 350 taxa (Bacteria), 83 taxa (Firmicutes), and 29 (Staphylococcus), respectively. Phylogenetic trees were constructed using maximum likelihood (ML) analysis provided by the program RAxML (version 8.2.9) ${ }^{48}$. The GAMMA Model of rate heterogeneity was used and all model parameters were estimated by RAxML. LG (Bacteria, Staphylococcus) and JTT (Firmicutes) with empirical base frequencies were determined to be the bestscoring (likelihood score) protein substitution model. Results were assessed using 
100 bootstrap replicates. The best tree was visualized using TreeGraph2 (version 2.14.0-771 beta) ${ }^{49}$.

\section{Occurrence of MpsAB and CA homologs based on PFam domains. The} occurrence of MpsAB and CA homologs were inferred based on PFam domains ${ }^{21}$ search from finished bacterial genomes in Integrated Microbial Genomes \& Microbiomes (IGM/G) database ${ }^{50}$. MpsA, MpsB, prokaryotic type-CA, and eukaryotic-type CA belongs to PFam00361, PFam10070, PFam00484, and PFam00194, respectively. Results were presented as the presence (if yes, the frequency it was found in a particular species) or absence of the respective PFam domains.

CA assays. CA assay was performed using purified $\mathrm{N}$-terminal strep-tagged MpsB protein. Two methods were used to detect CA activity, electrometric and colorimetric methods. The electrometric method refers to enzymatic assay of CA for Wilbur-Anderson Units (EC 4.2.1.1), as listed in the website of Sigma-Aldrich (document CR BIOT-MAJ-1013), based on cited references ${ }^{51-53}$. One WilburAnderson unit will cause the $\mathrm{pH}$ of a $20 \mathrm{mM}$ Trizma buffer to drop from 8.3 to 6.3 per minute at $0{ }^{\circ} \mathrm{C}$. The assay is briefly described here and the full protocol can be found on Sigma-Aldrich's website ${ }^{54}$. Assay buffer was prepared at $20 \mathrm{mM}$ using Trizma Base and purified water (Milli-Q) and the $\mathrm{pH}$ was adjusted to 8.3 with $2 \mathrm{~N}$ Sulfuric Acid. $\mathrm{CO}_{2}$ saturated solution was used as substrate and was prepared by placing a small piece of dry ice into cold Milli-Q water for at least $30 \mathrm{~min}$ prior to the experiment. CA enzyme standard solution was used as control. It was prepared fresh prior to the experiment by dissolving $2 \mathrm{mg}$ of CA from bovine erythrocytes lyophilized powder, $\geq 2000 \mathrm{~W}$-A units $\mathrm{mg}^{-1}$ protein (Sigma-Aldrich, Germany). Then the concentrated standard solution was diluted to $60-90 \mathrm{U} \mathrm{ml}^{-1}$. This concentration corresponds to a reaction time between 10 and $20 \mathrm{~s}$. The buffer reference standards ( $\mathrm{pH} 4,7$, and 10$)$ and $\mathrm{pH}$ electrode to equilibrate to $<3{ }^{\circ} \mathrm{C}$ before calibrating the $\mathrm{pH}$ meter with each of the reference buffer. All solutions and dram vials were kept cold on ice prior to use. To perform the blank reaction, $3 \mathrm{ml}$ of ice cold assay buffer and $0.05 \mathrm{ml}$ of ice cold purified water were pipetted into a dram vial with a micro stir bar. The temperature of the reaction mixture was checked with thermometer and proceed to the next step if the temperature was $<3^{\circ} \mathrm{C}$, by placing the $\mathrm{pH}$ electrode in the solution, with stirring. After the $\mathrm{pH}$ had reached $>8.5,2 \mathrm{ml}$ of ice cold $\mathrm{CO}_{2}$ saturated solution was added. The time required for the $\mathrm{pH}$ to change from 8.3 to 6.3 was recorded. This step was repeated a few times and once the blank time of $65 \mathrm{~s}$ was reached, the experiment proceeded immediately with CA control. For control, $3 \mathrm{ml}$ of ice cold assay buffer was added to the dram vial and if the temperature was $<3^{\circ} \mathrm{C}$ and subsequently when the $\mathrm{pH}>8.5,2 \mathrm{ml}$ of ice cold $\mathrm{CO}_{2}$ saturated solution was added to the reaction mixture. When the $\mathrm{pH}$ reached 8.4-8.5, $0.05 \mathrm{ml}$ of CA standard solution was added. The time required for the $\mathrm{pH}$ to change from 8.3 to 6.3 was recorded. If the time is not in the $10-20 \mathrm{~s}$ range, a more concentrated enzyme standard must be prepared. If the blank average time (from a minimum of five blank values) was in the range of 70-100 s, the experiment could proceed with purified MpsB protein. The steps for MpsB protein were the same as control, in which $0.05 \mathrm{ml}$ of MpsB was used instead of CA standard solution. The $\mathrm{CA} \mathrm{U} \mathrm{ml} l^{-1}$ is determined as:

$$
\text { Units } \mathrm{ml}^{-1} \text { enzyme }: \frac{\left(T_{\text {blank average }}-T_{\text {sample average }}\right) / \mathrm{df}}{\left(T_{\text {sample average }}\right)(0.05)}
$$

where $\mathrm{df}=$ Dilution factor

$T=$ Time (in seconds) required for the $\mathrm{pH}$ to change from 8.3 to 6.3 as per the Unit Definition

$0.05=$ Volume (in milliliter) of enzyme used

We found that this method is unreliable because of the difficulty in maintaining the temperature at $<3{ }^{\circ} \mathrm{C}$. Therefore, inconsistencies in the $\mathrm{pH}$ readings and subsequently the deviations in the time for the $\mathrm{pH}$ change from 8.3 to 6.3 resulted in inaccurate and non-reproducible readings. Therefore, we attempted another assay using colorimetric method. The colorimetric method to detect CA activity was performed according to previous studies ${ }^{20,55}$ with slight modifications. The assay was based on changing-pH/ dye indicator method using a Tecan Infinite M200 injector system. Briefly, two reaction buffers were prepared: (a) a buffer using phenol red as indicator $(200 \mathrm{mM}), 50 \mathrm{mM}$ HEPES, and $200 \mathrm{mM} \mathrm{Na}_{2} \mathrm{SO}_{4}$ at pH 6.5, 7, and 7.5, (b) a buffer using m-cresol purple as indicator $(200 \mathrm{mM}), 50 \mathrm{mM}$ TAPS, and $200 \mathrm{mM} \mathrm{Na}_{2} \mathrm{SO}_{4}$ at $\mathrm{pH} 7.5$ and 8.4. $\mathrm{CO}_{2}$ saturated water was prepared by placing a small piece of dry ice in Milli-Q water for at least 30 mins prior to the experiment. Purified MpsB protein was diluted in buffers and the reaction was initiated by adding an equivalent amount of $\mathrm{CO}_{2}$ saturated water with the Tecan injector system. The subsequent color changes of the $\mathrm{pH}$-sensitive dye indicators were monitored by Tecan microplate reader every $0.2 \mathrm{~s}$ for $1 \mathrm{~min}$ at $558 \mathrm{~nm}$ $(\mathrm{pH} 6.5-7.5)$ or $578 \mathrm{~nm}(\mathrm{pH} 7.5-8.4)$. All reactions were performed at room temperature $\left(\sim 25^{\circ} \mathrm{C}\right)$ and in a final volume of $1 \mathrm{ml}$. The CA activities (if any) of MpsB protein and positive control CA from bovine erythrocytes lyophilized powder (SigmaAldrich, Germany) were measured at final concentrations of 100 and $0.1 \mu \mathrm{g} \mathrm{ml}^{-1}$, respectively. A total of $50 \mathrm{mM}$ Tris- $\mathrm{HCl}$ at corresponding $\mathrm{pH}$ of the buffers was used as nonenzymatic controls. Due to the nondetectable CA activity, we repeated the assay with whole cell lysate of wild-type HG001, $\triangle m p s B$, and $\triangle m p s A B C$ with and without addition of NADH and finally with resting cell suspensions of the three strains. However, no detectable CA activity was observed.

Statistical analyses. Data are presented as mean values and SEM from at least three independent biological replicates unless specified otherwise. Data from the mouse experiment are presented as median values. Normal distributions were analyzed by Student's $t$-test. The larvae infection model was analyzed using Log-rank (Mantel-Cox) test. For the mouse infection experiments, two-tailed Mann-Whitneytest was employed to compare the difference of CFU counts in the organs between the mutant clones with the WT HG001. All statistical analyses were performed using GraphPad Prism 6.0 software. A $p$ value of $<0.05$ was considered to be statistically significant, with $n$ represents independent biological replicates.

Reporting summary. Further information on research design is available in the Nature Research Reporting Summary linked to this article.

\section{Data availability}

The source data underlying Figs. 1a, 2, 3, 5b \& c, 6 and Supplementary Figs. 2, 3, 6, and 7 are provided as a Source Data file. Other data supporting the findings of this work are available within the paper and its Supplementary Information files, or from the corresponding author upon request.

Received: 9 March 2019 Accepted: 16 July 2019

Published online: 09 August 2019

\section{References}

1. Gladstone, G. P., Fildes, P. \& Richardson, G. M. Carbon dioxide as an essential factor in the growth of bacteria. Br. J. Exp. Pathol. 16, 335-348 (1935).

2. Gill, P. \& Lobban, D. Carbon dioxide-dependent Staphylococcus aureus. Br. Med J. 2, 706 (1977).

3. Slifkin, M., Merkow, L. P., Kreuzberger, S. A., Engwall, C. \& Pardo, M. Characterization of CO 2 dependent microcolony variants of Staphylococcus aureus. Am. J. Clin. Pathol. 56, 584-592 (1971).

4. Wise, R. I. \& Spink, W. W. The influence of antibiotics on the origin of small colonies (G variants) of Micrococcus pyogenes var. aureus. J. Clin. Investig. 33, 1611-1622 (1954).

5. Proctor, R. A., van Langevelde, P., Kristjansson, M., Maslow, J. N. \& Arbeit, R. D. Persistent and relapsing infections associated with small-colony variants of Staphylococcus aureus. Clin. Infect. Dis. 20, 95-102 (1995).

6. Proctor, R. A. et al. Small colony variants: a pathogenic form of bacteria that facilitates persistent and recurrent infections. Nat. Rev. Microbiol. 4, 295-305 (2006).

7. Kahl, B. C., Becker, K. \& Loffler, B. Clinical Significance and Pathogenesis of Staphylococcal Small Colony Variants in Persistent Infections. Clin. Microbiol. Rev. 29, 401-427 (2016).

8. Garcia, L. G. et al. Intracellular forms of menadione-dependent small-colony variants of methicillin-resistant Staphylococcus aureus are hypersusceptible to beta-lactams in a THP-1 cell model due to cooperation between vacuolar acidic $\mathrm{pH}$ and oxidant species. J. Antimicrob. Chemother. 67, 2873-2881 (2012).

9. Kriegeskorte, A. et al. Thymidine-dependent Staphylococcus aureus smallcolony variants Are Induced by trimethoprim-sulfamethoxazole (SXT) and have increased fitness during SXT challenge. Antimicrob. Agents Chemother. 59, 7265-7272 (2015)

10. von Eiff, C. et al. A site-directed Staphylococcus aureus hemB mutant is a small-colony variant which persists intracellularly. J. Bacteriol. 179, 4706-4712 (1997).

11. Gomez-Gonzalez, C. et al. Clinical and molecular characteristics of infections with $\mathrm{CO}_{2}$-dependent small-colony variants of Staphylococcus aureus. J. Clin. Microbiol. 48, 2878-2884 (2010).

12. Mayer, S., Steffen, W., Steuber, J. \& Götz, F. The Staphylococcus aureus nuoLlike protein MpsA contributes to the generation of membrane potential. J. Bacteriol. 197, 794-806 (2015).

13. Mathiesen, C. \& Hagerhall, C. Transmembrane topology of the NuoL, M and $\mathrm{N}$ subunits of NADH:quinone oxidoreductase and their homologues among membrane-bound hydrogenases and bona fide antiporters. Biochim. Biophys. Acta 1556, 121-132 (2002).

14. Price, G. D. Inorganic carbon transporters of the cyanobacterial $\mathrm{CO}_{2}$ concentrating mechanism. Photosynth. Res. 109, 47-57 (2011).

15. Mangiapia, M. et al. Proteomic and mutant analysis of the $\mathrm{CO}_{2}$ concentrating mechanism of hydrothermal vent chemolithoautotroph Thiomicrospira crunogena. J. Bacteriol. 199, e00871-16 (2017).

16. Scott, K. M. et al. Diversity in $\mathrm{CO}_{2}$-concentrating mechanisms among chemolithoautotrophs from the genera Hydrogenovibrio, Thiomicrorhabdus, 
and Thiomicrospira, ubiquitous in sulfidic habitats worldwide. Appl. Environ. Microbiol. 85, e02096-18 (2019).

17. Herbert, S. et al. Repair of global regulators in Staphylococcus aureus 8325 and comparative analysis with other clinical isolates. Infect. Immun. 78, 2877-2889 (2010).

18. Merlin, C., Masters, M., McAteer, S. \& Coulson, A. Why is carbonic anhydrase essential to Escherichia coli? J. Bacteriol. 185, 6415-6424 (2003).

19. Fuchs, S. et al. AureoWiki The repository of the Staphylococcus aureus research and annotation community. Int J. Med. Microbiol. 308, 558-568 (2018).

20. Burghout, P. et al. Carbonic anhydrase is essential for Streptococcus pneumoniae growth in environmental ambient air. J. Bacteriol. 192, 4054-4062 (2010).

21. El-Gebali, S. et al. The Pfam protein families database in 2019. Nucleic Acids Res. 47, D427-D432 (2019).

22. Walker, J. E. The NADH:ubiquinone oxidoreductase (complex I) of respiratory chains. Q. Rev. Biophys. 25, 253-324 (1992).

23. Boron, W. F., Chen, L. \& Parker, M. D. Modular structure of sodium-coupled bicarbonate transporters. J. Exp. Biol. 212, 1697-1706 (2009).

24. Felder, R. A., Jose, P. A., Xu, P. \& Gildea, J. J. The renal sodium bicarbonate cotransporter NBCe2: is it a major contributor to sodium and $\mathrm{pH}$ homeostasis? Curr. Hypertens. Rep. 18, 71 (2016).

25. Romero, M. F. The electrogenic $\mathrm{Na}+/ \mathrm{HCO}_{3}{ }^{-}$cotransporter, NBC. JOP 2, 182-191 (2001).

26. Raven, J. A. \& Beardall, J. The ins and outs of $\mathrm{CO}_{2}$. J. Exp. Bot. 67, 1-13 (2016).

27. Raven, J. A., Beardall, J. \& Sanchez-Baracaldo, P. The possible evolution and future of $\mathrm{CO}_{2}$-concentrating mechanisms. J. Exp. Bot. 68, 3701-3716 (2017).

28. Shih, P. M. et al. Biochemical characterization of predicted Precambrian RuBisCO. Nat. Commun. 7, 10382 (2016).

29. Tabita, F. R., Hanson, T. E., Satagopan, S., Witte, B. H. \& Kreel, N. E. Phylogenetic and evolutionary relationships of RubisCO and the RubisCOlike proteins and the functional lessons provided by diverse molecular forms. Philos. Trans. R. Soc. Lond. B Biol. Sci. 363, 2629-2640 (2008).

30. Shibata, M. et al. Genes essential to sodium-dependent bicarbonate transport in cyanobacteria: function and phylogenetic analysis. J. Biol. Chem. 277, 18658-18664 (2002).

31. Price, G. D., Woodger, F. J., Badger, M. R., Howitt, S. M. \& Tucker, L. Identification of a SulP-type bicarbonate transporter in marine cyanobacteria. Proc. Natl. Acad. Sci. USA 101, 18228-18233 (2004).

32. Omata, T. et al. Identification of an ATP-binding cassette transporter involved in bicarbonate uptake in the cyanobacterium Synechococcus sp. strain PCC 7942. Proc. Natl. Acad. Sci. USA 96, 13571-13576 (1999).

33. Chou, C. Y., Yu, L. P. \& Tong, L. Crystal structure of biotin carboxylase in complex with substrates and implications for its catalytic mechanism. J. Biol. Chem. 284, 11690-11697 (2009).

34. Cherniack, N. S. \& Longobardo, G. S. Oxygen and carbon dioxide gas stores of the body. Physiol. Rev. 50, 196-243 (1970).

35. Lee, M. H. et al. A postinfluenza model of Staphylococcus aureus pneumonia. J. Infect. Dis. 201, 508-515 (2010).

36. Somerville, G. A. \& Proctor, R. A. Cultivation conditions and the diffusion of oxygen into culture media: the rationale for the flask-to-medium ratio in microbiology. BMC Microbiol. 13, 9 (2013).

37. Bae, T. \& Schneewind, O. Allelic replacement in Staphylococcus aureus with inducible counter-selection. Plasmid 55, 58-63 (2005).

38. Geiger, T. et al. The stringent response of Staphylococcus aureus and its impact on survival after phagocytosis through the induction of intracellular PSMs expression. PLoS Pathog. 8, e1003016 (2012).

39. Gibson, D. G. et al. Enzymatic assembly of DNA molecules up to several hundred kilobases. Nat. Methods 6, 343-345 (2009).

40. Monk I. R., Shah I. M., Xu M., Tan M. W., Foster T. J. Transforming the untransformable: application of direct transformation to manipulate genetically Staphylococcus aureus and Staphylococcus epidermidis. MBio. 3, e00277-11 (2012).

41. Ebner, P. et al. Excretion of cytoplasmic proteins (ECP) in Staphylococcus aureus. Mol. Microbiol. 97, 775-789 (2015).

42. Brückner, R. A series of shuttle vectors for Bacillus subtilis and Escherichia coli. Gene 122, 187-192 (1992).

43. Strauss, A., Thumm, G. \& Götz, F. Influence of Lif, the lysostaphin immunity factor, on acceptors of surface proteins and cell wall sorting efficiency in Staphylococcus carnosus. J. Bacteriol. 180, 4960-4962 (1998).

44. Diep, B. A. et al. Complete genome sequence of USA300, an epidemic clone of community-acquired meticillin-resistant Staphylococcus aureus. Lancet 367, 731-739 (2006).

45. Baba, T. et al. Genome and virulence determinants of high virulence community-acquired MRSA. Lancet 359, 1819-1827 (2002).
46. O'Leary, N. A. et al. Reference sequence (RefSeq) database at NCBI: current status, taxonomic expansion, and functional annotation. Nucleic Acids Res. $\mathbf{4 4}$ D733-D745 (2016).

47. Sievers, F. et al. Fast, scalable generation of high-quality protein multiple sequence alignments using Clustal Omega. Mol. Syst. Biol. 7, 539 (2011).

48. Stamatakis, A. RAxML version 8: a tool for phylogenetic analysis and postanalysis of large phylogenies. Bioinformatics 30, 1312-1313 (2014).

49. Stover, B. C. \& Muller, K. F. TreeGraph 2: combining and visualizing evidence from different phylogenetic analyses. BMC Bioinforma. 11, 7 (2010).

50. Chen, I. A. et al. IMG/M v.5.0: an integrated data management and comparative analysis system for microbial genomes and microbiomes. Nucleic Acids Res. 47, D666-D677 (2019).

51. Tripp, B. C., Smith, K. \& Ferry, J. G. Carbonic anhydrase: new insights for an ancient enzyme. J. Biol. Chem. 276, 48615-48618 (2001).

52. Wilbur, K. M. \& Anderson, N. G. Electrometric and colorimetric determination of carbonic anhydrase. J. Biol. Chem. 176, 147-154 (1948).

53. Worthington, C. C. (ed.) Worthington Enzyme Manual. pp 57-59, (Worthington Biochemical Corporation, Freehold, NJ, 1988).

54. Sigma Aldrich. Enzymatic Assay of Carbonic Anhydrase for Wilbur-Anderson units (EC 4.2.1.1). Sigma Aldrich. https://www.sigmaaldrich.com/technicaldocuments/protocols/biology/enzymatic-assay-of-carbonic-anhydrase.html

55. Khalifah, R. G. The carbon dioxide hydration activity of carbonic anhydrase. I. Stop-flow kinetic studies on the native human isoenzymes B and C. J. Biol. Chem. 246, 2561-2573 (1971).

\section{Acknowledgements}

This work was supported by grants from the Deutsche Forschungsgemeinschaft (DFG: SFB766, TRR34). S.H.F. received a PhD fellowship from the German Academic Exchange Service (DAAD) and Graduiertenkolleg (GRK) 1708. We thank Thomas Haug for his assistance with ${ }^{14} \mathrm{C}$ measurement. We would like to dedicate this article to the memory of Prof. Dr. Georg Peters and Prof. Dr. Olaf Schneewind, who passed away in August 2018 and May 2019, respectively. Both were wonderful colleagues whose passion and commitment to research greatly contributed to the field of microbiology, especially $S$. aureus biology.

\section{Author contributions}

F.G., P.E. and S.H.F. designed the study. S.R., P.E. and S.H.F. performed the cloning. T.H. and K.O. performed the mouse infection experiments. A.K.L., S.Z. and K.N. performed the phylogenetic and bioinformatics analyses. S.H.F. performed the rest of the experiments. F.G., S.H.F. and P.E. analyzed the data and wrote the paper.

\section{Additional information}

Supplementary Information accompanies this paper at https://doi.org/10.1038/s41467019-11547-5.

Competing interests: The authors declare no competing interests.

Reprints and permission information is available online at http://npg.nature.com/ reprintsandpermissions/

Peer review information: Nature Communications thanks Stephen Kidd, Greg Somerville and other anonymous reviewer(s) for their contribution to the peer review of this work

Publisher's note: Springer Nature remains neutral with regard to jurisdictional claims in published maps and institutional affiliations.



Open Access This article is licensed under a Creative Commons Attribution 4.0 International License, which permits use, sharing, adaptation, distribution and reproduction in any medium or format, as long as you give appropriate credit to the original author(s) and the source, provide a link to the Creative Commons license, and indicate if changes were made. The images or other third party material in this article are included in the article's Creative Commons license, unless indicated otherwise in a credit line to the material. If material is not included in the article's Creative Commons license and your intended use is not permitted by statutory regulation or exceeds the permitted use, you will need to obtain permission directly from the copyright holder. To view a copy of this license, visit http://creativecommons.org/ licenses/by/4.0/

(c) The Author(s) 2019 